\title{
AMBIENT SURGERY AND TANGENTIAL HOMOTOPY QUATERNIONIC PROJECTIVE SPACES
}

\author{
BY \\ DOUGLAS N. HERTZ
}

Introduction. In this paper the word manifold will always mean oriented compact $C^{\infty}$-manifold. Unless otherwise specified, all homology and cohomology is taken with integral coefficients, and for $M^{n}$ an $n$-manifold, $[M] \in H_{n}(M, \partial M)$ will denote the orientation class of $M$. A map $f: M \rightarrow N$ between $n$-manifolds is of degree +1 if $f_{*}([M])=[N]$.

We denote the quaternions by $Q$, and quaternionic projective $n$-space, which may be described as the collection of quaternionic lines in $Q^{n+1}$, by $Q P_{n}$. The underlying set of $Q P_{n}$ can also be described as

$$
\left\{\left(x_{1}, \ldots, x_{n+1}\right) \mid x_{i} \in Q \text { for } i=1, \ldots, n+1 ; \sum_{i=1}^{n+1}\left|x_{i}\right|^{2}=1\right\}
$$

modulo the equivalence relation $\left(x_{1}, \ldots, x_{n+1}\right) \sim\left(y_{1}, \ldots, y_{n+1}\right)$ if and only if for some $s \in Q,|s|=1,\left(x_{1}, \ldots, x_{n+1}\right)=s \cdot\left(y_{1}, \ldots, y_{n+1}\right)$. By $Q P_{n-1} \subset Q P_{n}$ we will always mean the copy of $Q P_{n-1}$ in $Q P_{n}$ defined by: the class of $\left(x_{1}, \ldots, x_{n+1}\right)$ is in $Q P_{n-1} \subset Q P_{n}$ if and only if $x_{n+1}=0 . Q P_{n}$ is given the structure of a manifold as in $[14, \S 20.3]$.

$\mathscr{S}^{4}$ will denote the unit 4-disc bundle over $Q P_{n}$ defined by the standard vector 4-bundle over $Q P_{n}$, whose total space is $\left\{(l, v) \mid l\right.$ a quaternionic line in $Q^{n+1}, v$ an element of $l\}$, and whose projection takes $(l, v)$ to $l$. $\mathscr{S}_{0}^{3}$ will denote the 3 -sphere bundle over $Q P_{n}$ associated with $\mathscr{S}^{4}$. The group of $\mathscr{S}_{0}^{3}$ may be reduced to $S^{3}=$ the multiplicative group of unit length quaternions, and, since the total space of $\mathscr{S}_{0}^{3}$ over $Q P_{n}$ is $S^{4 n+3}$, we see $\mathscr{S}_{0}^{3}$ is the universal bundle with fiber $S^{3}$ and group the multiplicative group of unit length quaternions for dimensions less than or equal to $4 n+2$, see $[14, \S 19]$.

Given a manifold $M$ with submanifold $N$, we denote the normal disc bundle of $N$ in $M$ by $\nu(N \subset M)$, and we identify a tubular neighborhood of $N$ in $M$ with the total space of $\nu(N \subset M) . \tau(M)$ will denote the tangent bundle of $M$, and $0_{M}^{n}$ the trivial vector $n$-bundle over $M$. A map between manifolds $f: R \rightarrow S$ is called tangential if for some integers $k, l, f^{*}(\tau(S)) \oplus 0_{R}^{k} \approx \tau(R) \oplus 0_{R}^{l}$.

Statement of results. The first section of this paper is devoted to a discussion of surgery [6], [8], [12]. In particular, we show that in special cases one can do surgery

Received by the editors March 7, 1968 and, in revised form, April 30, 1969.

Copyright (C) 1969, American Mathematical Society 
to a submanifold $N$ of a manifold $M$ so that the modified manifold is also a submanifold of $M$. Using this technique we prove

THEOREM 1. Let $P^{m-l}$ be a simply connected submanifold of a simply connected manifold $Q^{m}$, where $l \geqq 3, m-l \geqq 5$, and $m-l$ congruent to 0,1 , or 3 modulo 4 . Suppose $P, Q$, and $M^{m}$ are manifolds without boundary and that $f: M^{m} \rightarrow Q^{m}$ is a degree +1 tangential homotopy equivalence. Then $f$ is homotopic to a map $f^{\prime}: M \rightarrow Q$ which is differentiable and transverse regular over $P \subset Q$ and such that

is a homotopy equivalence.

$$
f^{\prime} \mid f^{\prime-1}(P): f^{\prime-1}(P) \rightarrow P
$$

We also prove a uniqueness theorem for the $m-l \equiv 0$ modulo 4 case:

Theorem 2. Let $P$ and $Q$ be as in Theorem 1 , with $m-l \equiv 0 \bmod 4$. Assume $M^{m+1}$ is an h-cobordism between $M_{1}^{m}$ and $M_{2}^{m}$. Let $f: M^{m+1} \rightarrow Q$ be a tangential homotopy equivalence which is differentiable and transverse regular over $P \subset Q$. Let $N_{i}=f^{-1}(P) \cap M_{i}$ for $i=1,2$. Assume further that $f \mid N_{i}: N_{i} \rightarrow P$ is a homotopy equivalence for $i=1,2$. Then $f$ is homotopic to a map $f^{\prime}: M^{m+1} \rightarrow Q$ such that $f^{\prime}\left|M_{1}^{m} \cup M_{2}^{m}=f\right| M_{1}^{m} \cup M_{2}^{m}$, and such that $f^{\prime}$ is differentiable and transverse regular over $P \subset Q$ and $f^{\prime} \mid f^{-1}(P): f^{\prime-1}(P) \rightarrow P$ is a homotopy equivalence so that $f^{-1}(P)$ is an h-cobordism between $N_{1}$ and $N_{2}$.

In $\S 2$ we apply the results of the first section to the study of manifolds of the same tangential homotopy type as $Q P_{n}$.

Definition. For $n \geqq 2, \theta\left(Q P_{n}\right)$ is the set of equivalence classes of pairs $(M, f)$ where $M$ is a $4 n$-manifold without boundary and $f: M \rightarrow Q P_{n}$ is a tangential homotopy equivalence of degree +1 under the relation: $(M, f) \sim(N, g)$ if and only if $M$ and $N$ are $h$-cobordant under a cobordism $C$ such that there is a tangential homotopy equivalence $h: C \rightarrow Q P_{n}$ which restricts to $f$ and $g$ on the proper boundary components.

Definition. $\theta_{n}$ is the group of homotopy $n$-spheres under the equivalence relation of $h$-cobordism, as discussed in [6]. We will see that $\theta_{4 n}$ acts as a group on $\theta\left(Q P_{n}\right)$ by connected sum for $n \geqq 2$.

Proofs that the following two definitions are independent of the choices made are given in $\S 2$. They depend on the theorems of $\S 1$.

Definition. Given $\alpha \in \theta\left(Q P_{n}\right)$, we will define $r(\alpha) \in \theta\left(Q P_{n-1}\right)$ by choosing a representative element $(M, f)$ for $\alpha$ such that $f: M \rightarrow Q P_{n}$ is differentiable and transverse regular over $Q P_{n-1} \subset Q P_{n}$ and such that $f \mid f^{-1}\left(Q P_{n-1}\right): f^{-1}\left(Q P_{n-1}\right) \rightarrow$ $Q P_{n-1}$ is a tangential homotopy equivalence, and setting

$$
r(\alpha)=\left[\left(f^{-1}\left(Q P_{n-1}\right), f \mid f^{-1}\left(Q P_{n-1}\right)\right)\right] .
$$

Definition. Given $\gamma \in \theta\left(Q P_{n-1}\right)$ we will define $h(\gamma) \in \theta_{4 n-1}$ as follows: choose a representative $(N, g)$ for $\gamma$ such that $g: N \rightarrow Q P_{n-1}$ is differentiable. Then $g^{*}\left(\mathscr{S}_{0}^{3}\right)$ is a homotopy sphere. We define $h(\gamma)=\left[g^{*}\left(\mathscr{S}_{0}^{3}\right)\right] \in \theta_{4 n-1}$. 
If for $\lambda \in \theta_{4 n}$ we let $c(\lambda)=\lambda \cdot\left[\left(Q P_{n}\right.\right.$, identity)], where the dot indicates the group action of $\theta_{4 n}$ in $\theta\left(Q P_{n}\right)$, we obtain a sequence

In $\S 2$ we prove

$$
\theta_{4 n} \stackrel{c}{\longrightarrow} \theta\left(Q P_{n}\right) \stackrel{r}{\longrightarrow} \theta\left(Q P_{n-1}\right) \stackrel{h}{\longrightarrow} \theta_{4 n-1} .
$$

THEOREM 3. (1) image of $r=$ kernel of $h$,

(2) if $r(\alpha)=r(\beta)$, then $\alpha=\lambda \cdot \beta$ for some $\lambda \in \theta_{4 n}$.

This theorem gives us an inductive geometric procedure whereby representatives for all elements of $\theta\left(Q P_{n}\right)$ may be constructed from elements of $\theta\left(Q P_{n-1}\right)$. We conclude $\$ 2$ with this construction.

In $\S 3$ we investigate $\theta\left(Q P_{2}\right)$ as a starting point for inductions. We prove

THEOREM 4. $\theta\left(Q P_{2}\right)$ contains at most two elements, with representatives given by $\left(Q P_{2}\right.$, identity) and $\left(Q P_{2} \# \Sigma^{8}, \mathrm{id}^{\prime}\right)$, where $\Sigma^{8}$ is the nonstandard homotopy 8-sphere,

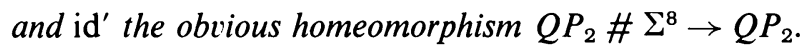

Note. This and Theorem 3 together imply that the number of elements in $\theta\left(Q P_{n}\right)$ is finite for all $n \geqq 2$, since $\theta_{n}$ is finite for all $n \geqq 8$ [6].

Definition. A map between manifolds, $h: M \rightarrow N$, is induced by a combinatorial equicalence if for some $C^{\infty}$ triangulations $\tau_{1}: K_{1} \rightarrow M, \tau_{2}: K_{2} \rightarrow N$, and some combinatorial equivalence of simplicial complexes $c: K_{1} \rightarrow K_{2}, h=\tau_{2} \circ c \circ \tau_{1}^{-1}$.

The final result of this paper is

THEOREM 5. For $n \geqq 2$, any representative $(M, f)$ of an element of $\theta\left(Q P_{n}\right)$ has the property that $f: M \rightarrow Q P_{n}$ is homotopic to a map $h: M \rightarrow Q P_{n}$ induced by a combinatorial equivalence. Thus, for $n \geqq 2$, all $4 n$-manifolds of the tangential homotopy type of $Q P_{n}$ are combinatorially equivalent.

Note. The restriction to manifolds of the tangential homotopy type of $Q P_{n}$ is essential. Hsiang [4] has shown that for any $n \geqq 2$ there exist infinitely many distinct manifolds of the homotopy type of $Q P_{n}$ all with different rational Pontrjagin classes. These manifolds are all combinatorially distinct.

This paper was essentially the author's doctoral dissertation at Brandeis University. I would like to thank A. Vasquez, J. Levine, and particularly my advisor, E. H. Brown, Jr., for their aid in its preparation.

1. Ambient surgery. Corresponding to the assumptions of Theorems 1 and 2, there are two cases in which we will show that surgery can be done within an ambient manifold:

Case (a). $P^{m-l}$ a submanifold of $Q^{m}$ of codimension $l$ where $l \geqq 3, m-l \geqq 5$, and $m-l \not \equiv 2 \bmod 4$. Suppose $P$ and $Q$ are both simply connected, and that $P, Q$, and $M^{m}$ are manifolds without boundary. Let $f_{j}: M \rightarrow Q$ be a degree +1 tangential homotopy equivalence. By the work of Thom [16] we can deform $f$ by a homotopy 
so that it becomes differentiable and transverse regular over $P \subset Q$. In this case we will do surgery to $f^{-1}(P)$ to prove that $f$ can be deformed by a series of homotopies to a map $f^{\prime}: M \rightarrow Q$ such that $f^{\prime}$ is transverse regular over $P \subset Q$ and

$$
f^{\prime} \mid f^{\prime-1}(P): f^{\prime-1}(P) \rightarrow P
$$

is a homotopy equivalence.

Case (b). Assume $P^{m-l}$ and $Q^{m}$ are as in Case (a), but with $m-l \equiv 0 \bmod 4$. Suppose $M^{m+1}$ is an $h$-cobordism between $M_{1}^{m}$ and $M_{2}^{m}$ and $f: M^{m+1} \rightarrow Q$ is a tangential homotopy equivalence. Then $f \mid M_{i}^{m}: M_{i}^{m} \rightarrow Q$ is a homotopy equivalence for $i=1,2$. Again we may up to homotopy assume $f$ differentiable and transverse regular over $P \subset Q$. We assume $f$ is of this form, and further that if $N_{i}=f^{-1}(P) \cap M_{i}$ then $f \mid N_{i}: N_{i} \rightarrow P$ is a homotopy equivalence for $i=1,2$. In this case we will do surgery to $f^{-1}(P)$ to prove that $f: M^{m+1} \rightarrow Q$ can be deformed by a series of homotopies, all of which are stationary on $\partial M^{m+1}$, to a map $f^{\prime}: M^{m+1} \rightarrow Q$ which is differentiable and transverse regular over $P \subset Q$ and such that $f^{\prime-1}(P)$ is an $h$-cobordism between $N_{1}$ and $N_{2}$.

Recall the following lemma from [16, p. 67].

Lemma 1.1. Given $f: M^{m} \rightarrow Q$ differentiable and transverse regular over $P \subset Q$ as in Case (a), if we put $N=f^{-1}(P)$ then $f \mid N: N \rightarrow P$ is a map of degree \pm 1 , and thus $N$ may be oriented so that $f \mid N$ is a map of degree +1 .

In dealing with both Cases (a) and (b) from now on, we will assume $f: M \rightarrow Q$ is differentiable and transverse regular over $P \subset Q$, and denote $f^{-1}(P)$ by $N$ and $f \mid N: N \rightarrow P$ by $g: N \rightarrow P$.

We now make the inductive assumption that $g: N \rightarrow P$ is such that $g_{*}: H_{r}(N) \rightarrow$ $H_{r}(P)$ is an isomorphism for all $r<k$, where $0 \leqq k \leqq(m-l) / 2$. We will want to do surgery to $N$ to modify $H_{k}(N)$. By the transversality of $f$ to $P \subset Q$, we have $\nu(N \subset M)$ $\approx g^{*}(\nu(P \subset Q))$ and thus the assumption that $f$ is tangential implies $g: N \rightarrow P$ is tangential. In Case (a) Lemma 1.1 implies $g$ is a map of degree +1 , so by [12] we see $g_{*}: H_{k}(N) \rightarrow H_{k}(P)$ is onto, and all classes in the kernel of this map are spherical.

For Case (b), the map $g_{*}: H_{i}(N) \rightarrow H_{i}(P)$ is always onto, and since $g_{*}: \pi_{i}(N) \rightarrow$ $\pi_{i}(P)$ is an isomorphism for all $i<k$, we have that all classes in the kernel of $g_{*}: H_{k}(N) \rightarrow H_{k}(P)$ are spherical see [12].

In either case, let $\alpha \in H_{k}(N)$ be an element of kernel $\left(g_{*}: H_{k}(N) \rightarrow H_{k}(P)\right)$. Since $\alpha$ is spherical and $k \leqq(m-l) / 2, m-l \geqq 5$, we may represent $\alpha$ by an imbedding $i: S^{k} \rightarrow N$. In Case (b) it is clear we may choose $i$ so that $i\left(S^{k}\right) \cap \partial N=\varnothing$. We will assume that $\alpha$ is so represented, and further that $\nu\left(i\left(S^{k}\right) \subset N\right)$ is trivial. It should be noted that $i: S^{k} \rightarrow N$ is nullhomotopic in $M$, and thus

$$
\tau(M)\left|i\left(S^{k}\right) \approx 0^{m} \approx \nu\left(i\left(S^{k}\right) \subset N\right) \oplus \nu(N \subset M)\right| i\left(S^{k}\right) .
$$

Hence $\nu\left(i\left(S^{k}\right) \subset N\right)$ is stably trivial, and this further condition is no restriction for $k<(m-l) / 2$. In the middle dimension $k=(m-l) / 2$ we must show that kernel 
$\left(g_{*}: H_{k}(N) \rightarrow H_{k}(P)\right)$ can be reduced to zero by doing surgery only on classes $\alpha \in H_{k}(N)$ which satisfy this additional condition.

The idea of the following work is to attach a $(k+1)$-disc to $N$ in $M$ via the map $i$, and, by thickening this disc, to get a spherical modification of $N$ to a new submanifold $N^{\prime}$ of $M$ and to deform the map $f: M \rightarrow Q$ by a homotopy to a map $f_{1}$ such that $f_{1}^{-1}(P)=N^{\prime}$. The following lemma is readily seen, and its proof is omitted.

Lemma 1.2. The map $f: M \rightarrow Q$ is homotopic to a map $f_{1}: M \rightarrow Q$ such that $f_{1}^{-1}(P)=N=f^{-1}(P), f_{1}$ is differentiable and transverse regular over $P \subset Q$, and $f_{1}$ maps a neighborhood of $i\left(S^{k}\right)$ in $N$ to a point $p \in P$.

Henceforth we will assume $f: M \rightarrow Q$ has the properties described for $f_{1}$ in Lemma 1.2.

By the transversality of $f$ we know $\nu(N \subset M) \approx g^{*}(\nu(P \subset Q))$, so, by choosing a framing for $\nu(P \subset Q) \mid p$, where $p=f \circ i\left(S^{k}\right)$, we get a framing $\nu_{1}, \ldots, v_{l}$ for $\nu(N \subset M) \mid i\left(S^{k}\right)$. We identify $\nu(N \subset M)$ with a tubular neighborhood of $N$ in $M$ and push $i\left(S^{k}\right)$ out into $M-N$ by a map $i_{1}: S^{k} \times I \rightarrow M$ defined by $i_{1}(x, \tau)=$ $\left(i(x), \tau \cdot \nu_{1}\right)$. Since $f \circ i: S^{k} \rightarrow P$ is nullhomotopic and $f: M \rightarrow Q$ is a homotopy equivalence, the class of $i_{1} \mid\left(S^{k} \times(1)\right)$ is null in $\pi_{k}(M)$. The following crucial proposition asserts this holds also in $M-N$.

Proposition 1.1. $i_{1} \mid\left(S^{k} \times(1)\right)$ is a nullhomotopic imbedding of $S^{k}$ in $M-N$.

Proof. The proof of this proposition will be in several parts.

(1) If $k=0$, we have $S^{k}$ is just $S^{0}=2$ points. Since $N \cap i_{1}\left(S^{0} \times(1)\right)=\varnothing$, and $M$ is path connected, we may choose a differentiable arc $h: I \rightarrow M$ connecting the points of $i_{1}\left(S^{0} \times(1)\right)$, transverse regular over $N$. In Case (b), $i_{1}\left(S^{0} \times(1)\right) \cap \partial M$ can be assumed empty, and we may choose $h: I \rightarrow M$ so that $h(I) \cap \partial M=\varnothing$. The condition that $h$ is transverse to $N$ implies $h(I) \cap N=\varnothing$ since codimension of $N$ in $M$ is $l>1$, so $i_{1}\left(S^{0} \times(1)\right)$ is nullhomotopic in $M-N$.

(2) For $k=1$ a similar proof applies. That is, we take a map $h: D^{2} \rightarrow M$ such that $h\left|\partial D^{2}=i_{1}\right|\left(S^{1} \times(1)\right)$ which is transverse to $N$. As above $h$ can be chosen so that $h\left(D^{2}\right) \cap \partial M=\varnothing$ in case (b). Transversality again implies $h\left(D^{2}\right) \cap N=\varnothing$ since codimension of $N$ in $M$ is $l>2$.

(3) For $k \geqq 2$, the following lemma is applied to show the proposition. In applying the lemma, we note that $f \circ i_{1} \mid\left(S^{k} \times(1)\right)$ is clearly nullhomotopic in $Q-P$.

Lemma 1.3. Suppose $k \geqq 2$ and $g_{*}: H_{p}(N) \rightarrow H_{p}(P)$ is an isomorphism for all $p<k$. Then if $f^{\prime}=f \mid M-N, f_{*}^{\prime}: \pi_{p}(M-N) \rightarrow \pi_{p}(Q-P)$ is an isomorphism for $p \leqq k+l-2$.

Proof. We first prove this for Case (a). $f$ is transverse regular over $P \subset Q$, so by [16] we may choose a tubular neighborhood $T(P)$ of $P$ in $Q$ so that $f^{-1}(T(P)) \equiv T(N)$ 
is a tubular neighborhood of $N$ in $M$. We define $M^{\prime}=M$-interior of $T(N)$; $Q^{\prime}=Q$-interior of $T(P)$. Then $M^{\prime}$ and $Q^{\prime}$ are manifolds with boundary, and $H_{p}(M-N) \approx H_{p}\left(M^{\prime}\right), H_{p}(Q-P) \approx H_{p}\left(Q^{\prime}\right)$. Consider the diagram:

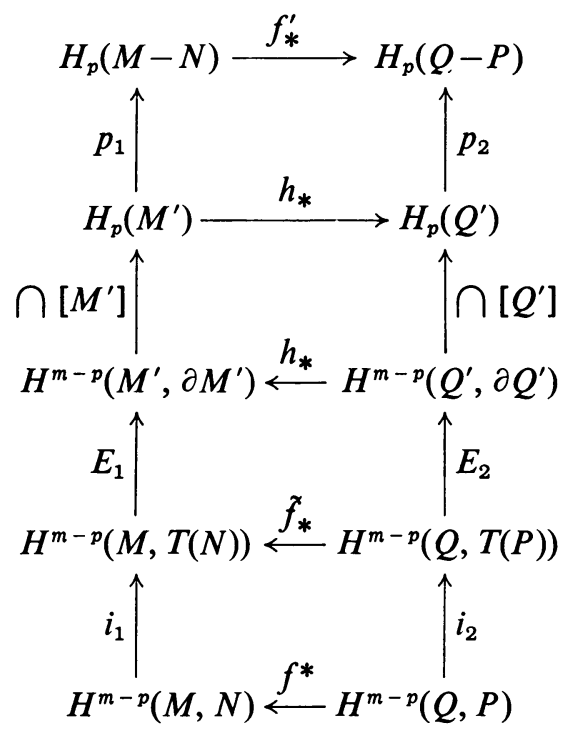

where the maps are the obvious inclusions or restrictions of $f$. All of the vertical maps are isomorphisms. By naturality of the cap product and the fact that $h_{*}$ has degree $+1, h_{*} \circ\left(\bigcap\left[M^{\prime}\right]\right) \circ h^{*}=\bigcap\left[Q^{\prime}\right]$. Hence $f^{*}$ is a monomorphism, and $f_{*}^{\prime}$ is an epimorphism. Further, if $f^{*}$ is an isomorphism, then so is $f_{*}^{\prime}$.

Consider the commutative diagram:

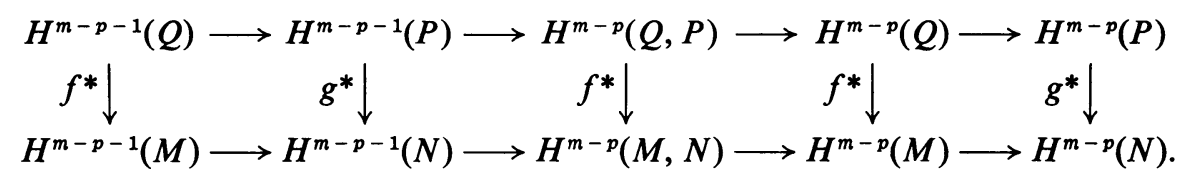

From this and the 5-lemma we conclude that $f^{*}: H^{m-p}(Q, P) \rightarrow H^{m-p}(M, N)$ is an isomorphism whenever both $g^{*}: H^{m-p-1}(P) \rightarrow H^{m-p-1}(N)$ and $g^{*}: H^{m-p}(P) \rightarrow$ $H^{m-p}(N)$ are.

The lemma of Novikov [7, p. 10] implies these conditions hold if

$$
g_{*}: H_{p+1-l}(N) \rightarrow H_{p+1-l}(P) \text { and } g_{*}: H_{p-l}(N) \rightarrow H_{p-l}(P)
$$

are isomorphisms. Thus $f^{*}: H^{m-p}(Q, P) \rightarrow H^{m-p}(M, N)$, and hence

$$
f_{*}^{\prime}: H_{p}(M-N) \rightarrow H_{p}(Q-P),
$$

is an isomorphism for $p+1-l<k$, or $p \leqq k+l-2$.

Since $k \geqq 2, \pi_{1}(N) \approx \pi_{1}(P)=0$, and, by assumption, $\pi_{1}(M) \approx \pi_{1}(Q)=0$, so by Van Kampen's Theorem, $\pi_{1}(M-N) \approx \pi_{1}(Q-P)=0$. We have shown

$$
f_{*}^{\prime}: H_{p}(M-N) \rightarrow H_{p}(Q-P)
$$


is onto for all $p$, so by Whitehead's Theorem we now see $f_{*}^{\prime}: \pi_{p}(M-N) \rightarrow \pi_{p}(Q-P)$ is an isomorphism for all $p \leqq k+l-2$. This concludes the proof of Lemma 1.3 and of Proposition 1.1 for Case (a).

Recall that in Case (b) $M$ is assumed to be an $h$-cobordism between $M_{1}$ and $M_{2}$, $N_{1}=N \cap M_{1}$ and $f \mid M_{1}: M_{1} \rightarrow Q$ and $f \mid M_{2}: M_{2} \rightarrow Q$ are both assumed to be homotopy equivalences.

By the lemma for Case (a), $f_{*}^{\prime}: H_{p}\left(M_{1}-N_{1}\right) \rightarrow H_{p}(Q-P)$ is an isomorphism for all $p$. Hence $f_{*}^{\prime}: H_{p}(M-N) \rightarrow H_{p}(Q-P)$ is onto for all $p$, and inclusion ${ }_{*}$ : $H_{p}\left(M_{1}-N_{1}\right) \rightarrow H_{p}(M-N)$ is one-to-one for all $p$. Thus $f_{*}^{\prime}: H_{p}(M-N) \rightarrow H_{p}(Q-P)$ is an isomorphism if inclusion ${ }_{*}: H_{p}\left(M_{1}-N_{1}\right) \rightarrow H_{p}(M-N)$ is onto.

Choose disc bundle structures on tubular neighborhoods $T\left(N_{1}\right)$ and $T(N)$ of $N_{1}$ in $M_{1}$ and $N$ in $M$ respectively so that the inclusion gives a bundle map $T\left(N_{1}\right) \rightarrow$ $T(N)$. Then the naturality of the Thom isomorphism implies that if

$$
\operatorname{incl}_{*}: H_{p}\left(N_{1}\right) \rightarrow H_{p}(N)
$$

is an isomorphism, then so is $\operatorname{incl}_{*}: H_{p+l}\left(T\left(N_{1}\right), \partial T\left(N_{1}\right)\right) \rightarrow H_{p+l}(T(N), \partial T(N))$.

By excision, $H_{p+l}\left(T\left(N_{1}\right), \partial T\left(N_{1}\right)\right) \approx H_{p+l}\left(M_{1}, M_{1}-N_{1}\right)$ and $H_{p+l}(T(N), \partial T(N))$ $\approx H_{p+l}(M, M-N)$. Also, if $g_{*}: H_{p}(N) \rightarrow H_{p}(P)$ is an isomorphism so is incl ${ }_{*}$ : $H_{p}\left(N_{1}\right) \rightarrow H_{p}(N)$, so for $p<k$ we have isomorphisms as indicated in the following diagram:

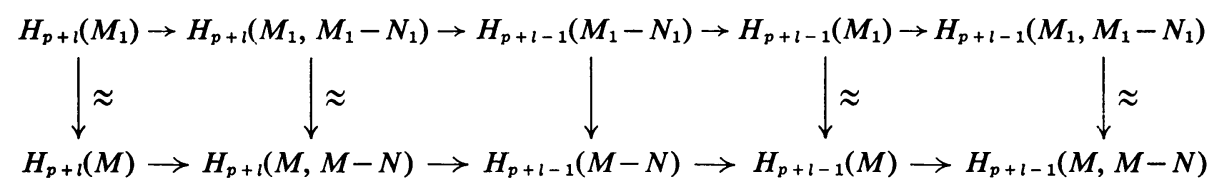

By the 5-lemma we conclude incl ${ }_{*}: H_{p+l-1}\left(M_{1}-N_{1}\right) \rightarrow H_{p+l-1}(M-N)$ is an isomorphism for $p<k$, and then $\operatorname{incl}_{*}: H_{i}\left(M_{1}-N_{1}\right) \rightarrow H_{i}(M-N)$ is an isomorphism for $i \leqq k+l-2$. Thus we see $f_{*}^{\prime}: H_{p}(M-N) \rightarrow H_{p}(Q-P)$ is an isomorphism for $p \leqq k+l-2$, and, as in the proof of Case (a), $M-N$ and $Q-P$ are simply connected, so we again apply Whitehead's Theorem to show $f_{*}^{\prime}: \pi_{p}(M-N) \rightarrow \pi_{p}(Q-P)$ is an isomorphism for $p \leqq k+l-2$. This completes the proof of Lemma 1.3 and of Proposition 1.1.

We now identify $\left\{x \in D^{k+1}|| x \mid \geqq 1 / 2\right\}$ with $S^{k} \times I$, and define a map

$$
h:\left\{x \in D^{k+1}|| x \mid \geqq 1 / 2\right\} \rightarrow M
$$

to be this identification composed with the above defined map $i_{1}$. Since $f$ is transverse to $P \subset Q$ and carries $i_{1}\left(S^{k} \times(0)\right)$ to $p \in P, f \circ h$ maps $S_{1 / 2}^{k}=\left\{x \in D^{k+1}|| x \mid=1 / 2\right\}$ to a single point $x \in Q-P$. Denote $\left\{x \in D^{k+1}|| x \mid \leqq 1 / 2\right\}$ by $D_{1 / 2}^{k+1}$ so that

$$
\partial D_{1 / 2}^{k+1}=S_{1 / 2}^{k} \text {. }
$$

LEMMA 1.4. There is a map $h^{\prime}: D_{1 / 2}^{k+1} \rightarrow M-N$ such that $h^{\prime}\left|S_{1 / 2}^{k}=h\right| S_{1 / 2}^{k}$, and $f \circ h^{\prime}:\left(D_{1 / 2}^{k+1}, S_{1 / 2}^{k}\right) \rightarrow(Q-P, x)$ is nullhomotopic. Thus $f: M \rightarrow Q$ can be deformed by a homotopy so that we may assume $f \circ h^{\prime}\left(D_{1 / 2}^{k+1}\right)=x$. 
Proof. Consider the diagram:

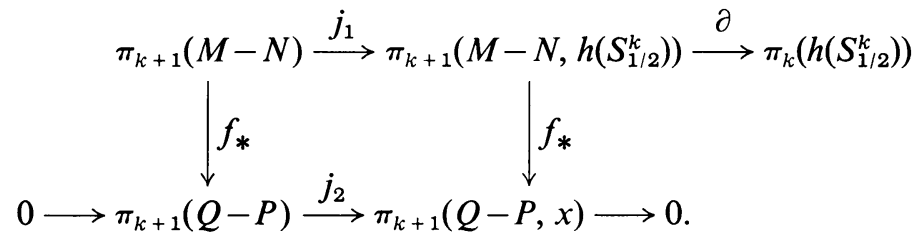

$h \mid S_{1 / 2}^{k}: S_{1 / 2}^{k} \rightarrow M-N$ is nullhomotopic, so there is a map $h_{1}: D_{1 / 2}^{k+1} \rightarrow M-N$ which extends $h$. Suppose $h_{1}:\left(D_{1 / 2}^{k+1}, S_{1 / 2}^{k}\right) \rightarrow\left(M-N, h\left(S_{1 / 2}^{k}\right)\right)$ represents an element $\alpha \in \pi_{k+1}\left(M-N, h\left(S_{1 / 2}^{k}\right)\right)$. Let $\gamma=j_{1} \circ\left(j_{2} f_{*}\right)^{-1} \circ f_{*}(\alpha)$. Then a representative of the class $\alpha+(-\gamma)$ defines a map $h^{\prime}: D_{1 / 2}^{k+1} \rightarrow M-N$ which agrees with $h$ on $S_{1 / 2}^{k}$ such that $f \circ h^{\prime}:\left(D_{1 / 2}^{k+1}, S_{1 / 2}^{k}\right) \rightarrow(Q-P, x)$ is nullhomotopic.

We may choose $h^{\prime}$ within its class to be an imbedding such that the map defined on $D^{k+1}$ by $h^{\prime}$ on $D_{1 / 2}^{k+1}$ and $h$ on $\left\{x \in D^{k+1}|| x \mid \geqq 1 / 2\right\}$ is an imbedding [1, Theorem 4.1]. Also, we may now deform $f$ by a homotopy in a tubular neighborhood of $h^{\prime}\left(D_{1 / 2}^{k+1}\right)$ so that $f \circ h^{\prime}\left(D_{1 /}^{k+1}\right)=x \in Q-P$.

We will henceforth denote the map $D^{k+1} \rightarrow M$ constructed in Lemma 1.4 by the letter $h$, and will assume that $f: M \rightarrow Q$ has been put into the form described in that lemma, and in Lemma 1.2.

We have assumed that $\nu\left(i\left(S^{k}\right) \subset N\right)$ is trivial, so $\nu\left(h\left(D^{k+1} \subset M\right)\right) \mid h\left(S^{k}\right)$ is the sum of two bundles, $\eta_{1}$ and $\eta_{2}$, where $\eta_{1}$ is the $(l-1)$-dimensional trivial bundle given by $\nu_{2}, \ldots, \nu_{l}$, that is the bundle which results when we split

$$
\nu_{1}=\nu\left(h\left(S^{k}\right) \subset h\left(D^{k+1}\right)\right)
$$

off $\nu(N \subset M) \mid h\left(S^{k}\right)$, and $\eta_{2}$ is an $(m-l-k)$-dimensional trivial bundle in Case (a) and an $(m+1-l-k)$-dimensional trivial bundle in Case (b). $\eta_{1}$ is, of course, already framed by our choice of $\nu_{2}, \ldots, \nu_{l}$, pulled back by $f$ from $\nu(P \subset Q) \mid p$.

LEMMA 1.5. With notation as above, there is a framing of $\nu\left(h\left(D^{k+1}\right) \subset M\right)$ such that restricted to $h\left(S^{k}\right)$ it gives the required framing $\nu_{2}, \ldots, \nu_{l}$ of $\eta_{1}$, and some framing of $\eta_{2}$.

Proof. Framings of $\nu\left(h\left(D^{k+1}\right) \subset M\right) \mid h\left(S^{k}\right)$ are represented by elements of $\pi_{k}(S O(m-k-1))$ in Case (a), and elements of $\pi_{k}(S O(m-k))$ in Case (b), and extend over $h\left(D^{k+1}\right)$ if and only if the representative homotopy class is zero. Similarly, elements of $\pi_{k}(S O(m-l-k))$ in Case (a) and $\pi_{k}(S O(m+1-l-k))$ in Case (b) represent framings of $\eta_{2}=\nu\left(h\left(S^{k}\right) \subset N\right)$. There is given a framing of $\eta_{1}=\nu(N \subset M) \mid h\left(S^{k}\right)$. Thus the problem of framing $\eta_{2}$ so that we get a framing of $\nu\left(h\left(D^{k+1}\right) \subset M\right) \mid h\left(S^{k}\right)$ which extends over $h\left(D^{k+1}\right)$ depends only on the map $\pi_{k}(S O(m-l-k)) \rightarrow \pi_{k}(S O(m-k-1))$ in Case (a) and on $\pi_{k}(S O(m+1-l-k)) \rightarrow$ $\pi_{k}(S O(m-k))$ in Case (b) induced by inclusion. If the map is onto then $\eta_{2}$ may be framed as needed. 
We see this by showing $\pi_{k}(S O(p)) \rightarrow \pi_{k}(S O(p+1))$ is onto for all relevant values of $p$. By Steenrod [14, p. 117] this map is onto whenever $k<p$. Since we have by assumption $k \leqq(m-l) / 2$, or $k<m-l-k+1$, the proof for Case (b) is complete. Also, for Case (a), if $m-l \equiv 1$ or $3 \bmod 4$, then $k<(m-l) / 2$, and $k<m-l-k$, so the proof is also complete in these cases.

Case (a), $m-l \equiv 0 \bmod 4$ remains, since we may have here $k=m-k-l$ or $k=(m-l) / 2$. Then $k$ is an even number, and in the fibration sequence

$$
\rightarrow \pi_{k+1}\left(S^{k}\right) \rightarrow \pi_{k}(S O(k)) \rightarrow \pi_{k}(S O(k+1)) \rightarrow \pi_{k}\left(S^{k}\right) \rightarrow \pi_{k-1}(S O(k)) \rightarrow
$$

it is known that $\pi_{k}\left(S^{k}\right) \rightarrow \pi_{k-1}(S O(k))$ is a monomorphism, so $\pi_{k}(S O(k) \rightarrow$ $\pi_{k}(S O(k+1))$ is onto, and we are done.

Note. In the above lemma a framing of $\eta_{2}$ which extends over $h\left(D^{k+1}\right)$ is found. In Case (a) for $m-l \equiv 3 \bmod 4$, we will later need the fact that there are many such framings from which we may choose. Lemma 1.5 shows the framing $\nu_{2}, \ldots, \nu_{l}$ of $\eta_{1}$ is represented by the zero element of $\pi_{k}\left(V_{m-k-1, l-1}\right)$. Different methods of extending this frame over $h\left(D^{k+1}\right)$ in $\nu\left(h\left(D^{k+1}\right) \subset M\right)$ are in 1-1 correspondence with elements of $\pi_{k+1}\left(V_{m-k-1, l-1}\right)$. In the fibration sequence

$$
\begin{aligned}
\pi_{k+1}(S O(m-k-1)) & \longrightarrow \pi_{k+1}\left(V_{m-k-1, l-1}\right) \stackrel{\partial}{\longrightarrow} \pi_{k}(S O(m-l-k)) \\
& \longrightarrow \pi_{k}(S O(m-k-1))
\end{aligned}
$$

we see $\pi_{k}(S O(m-k-1))$ is the stable group since $k<m-k-l \leqq m-k-3$, so elements in the image of the map $\partial$ are just the stably trivial elements of

$$
\pi_{k}(S O(m-l-k)) \text {. }
$$

Consequently, given any framing of $\eta_{2}$ which arises as in Lemma 1.5 for Case (a), $m-l \equiv 3 \bmod 4$, we may modify this frame by any stably trivial element of $\pi_{k}(S O(m-l-k))$ and still have a frame which arises as in that lemma. The author is grateful to J. Levine for pointing out this possibility. See [5].

We have now attached a disc $h\left(D^{k+1}\right)$ to $i\left(S^{k}\right)$ in $M$, and have framed

$$
\nu\left(h\left(D^{k+1}\right) \subset M\right)
$$

in such a way as to induce our given frame $\nu_{2}, \ldots, \nu_{l}$ on $\nu(N \subset M) \mid h\left(S^{k}\right)$ and some frame on $\nu\left(i\left(S^{k}\right) \subset N\right)=\eta_{2}$. If we look at those frames on $h\left(D^{k+1}\right)$ which restrict to $\eta_{2}$ on the boundary, and take a tubular neighborhood of these, we get an imbedding

$$
\begin{aligned}
\phi: D^{k+1} \times D^{m-l-k} & \rightarrow M^{m} \text { in Case (a), or } \\
\phi: D^{k+1} \times D^{m+1-l-k} & \rightarrow M^{m+1} \text { in Case (b) }
\end{aligned}
$$

where $\phi \mid\left(S^{k} \times(0)\right)$ represents the class $\alpha \in H_{k}(N)$ we wish to kill by surgery. The modified manifold $N^{\prime}$ we seek is given after suitable rounding of corners by

and by

$$
N-\text { int } \phi\left(S^{k} \times D^{m-l-k}\right) \cup \phi\left(D^{k+1} \times S^{m-l-k-1}\right) \text { in Case (a) }
$$

$$
N-\text { int } \phi\left(S^{k} \times D^{m+1-l-k}\right) \cup \phi\left(D^{k+1} \times S^{m-l-k}\right) \text { in Case (b). }
$$


We now want to find a homotopy of $f: M \rightarrow Q$ to a map $f_{1}: M \rightarrow Q$ which is differentiable and transverse regular over $P \subset Q$ and such that $f_{1}^{-1}(P)$ is "isotopic" to the modified manifold $N^{\prime}$ described above. Since bundle maps can be carried along over homotopies, we will have that $f_{1}$ is a tangential map, and so will have recovered the original conditions and be able to proceed inductively. Our method is similar to that of Haefliger [2].

Recall that $h: D^{k+1} \rightarrow M$ starts along the vector field $\nu_{1}$ in $v(N \subset M) \mid i\left(S^{k}\right)$. We extend this imbedding of $D^{k+1}$ in $M$ to an imbedding of $D_{2}^{k+1}=$ the $(k+1)$-disc of radius 2 in $M$ by mapping along the vector field $-v_{1}$ in a tubular neighborhood of $N$ in $M$. Denote the resulting map by $h: D_{2}^{k+1} \rightarrow M$. The framing we have chosen for $v\left(h\left(D^{k+1}\right) \subset M\right)$ extends over $\nu\left(h\left(D_{2}^{k+1}\right) \subset M\right)$.

In the lemmas above, we have modified the map $f$ so that $f$ maps a tubular neighborhood of $i\left(S^{k}\right)$ in $N$ to $p \in P$, is transverse to $P$, and $f \circ h \mid D_{1 / 2}^{k+1}$ has image a point $x \in Q-P$. It is now clear one can deform $f$ by a homotopy and choose coordinates for a tubular neighborhood of $h\left(D_{2}^{k+1}\right)$ in $M$ of the form $(x, y, z) \in D_{2}^{k+1}$ $\times D_{2}^{r} \times D^{l-1}$, where $r=m-l-k$ in Case (a) and $r=m+1-l-k$ in Case (b), such that on this neighborhood the map $f$ is defined by the following composition:

Let $s_{1}: R \times D^{l-1} \rightarrow[-1,1] \times D^{l-1}$ be identity on $[-1,1] \times D^{l-1}, s_{1}(x, p)$ $=(-1, p)$ for $x \leqq-1$, and $s_{1}(x, p)=(1, p)$ for $x \geqq 1$.

$s_{2}:[-1,1] \times D^{l-1} \rightarrow D^{l}$ be a combinatorial equivalence defined by radial shrinking.

$s_{3}: D^{l} \approx$ fiber over $p \in P$ in a tubular neighborhood of $P$ in $Q$.

Then $f$ can be described as $(x, y, z) \mapsto s_{3} \circ s_{2} \circ s_{1}(-2|x|+2, z)$, where $|x|$ denotes the length of $x \in D_{2}^{k+1}$.

LEMMA 1.6. There is a map $r$ from the square $0 \leqq x, y \leqq 2$ in the plane to $R$ which is differentiable and transverse regular to $0 \in R$ and such that $r(2, y)=-2$ for all $y$, $r(x, 2)=-2 x+2$, and such that $r^{-1}(0)$ is a connected 1-dimensional submanifold of the square containing the segments $\{0 \leqq x \leqq 1 / 4, y=1 / 4\},\{x=1,1 \leqq y \leqq 2\}$ and such that

$$
r^{-1}(0) \cap(\{1 \leqq y \leqq 2\} \cup\{1 \leqq x \leqq 2\} \cup\{0 \leqq y \leqq 1 / 4\})
$$

is just these segments. Further, one can choose such a function $r$ so that $r(x, y)=-2$ for $y \leqq 1 / 10$, and $r(x, y) \geqq 1.1$ for $(x, y) \in\{x \leqq 1 / 10, y \geqq 1.8\}$, and so that $r$ is a function of $y$ only on the set $\{x \leqq 1 / 10, y \leqq 1.8\}$.

Proof. Define a function in polar coordinates in this region of the plane by $g(r, \theta)=r^{2} \cdot\left(\cos ^{2}(\theta-\pi / 4)-\sin ^{2}(\theta-\pi / 4)\right)+c\left(r^{2}\right)$ where $c:[0,4] \rightarrow R$ is a $C^{\infty}$ decreasing map with $c(0)=1, c\left(\frac{1}{2}\right)=\frac{1}{2}$, and $c(\tau)=0$ for $\tau \geqq \frac{3}{4}$. Let $h_{1}(x, y)$ be this same function in ordinary orthogonal coordinates.

Let $h_{2}(x, y)=-h_{1}(x-1, y-1 / 4)$, and $h_{3}(x, y)=p(x) \cdot h_{2}(x, y)+p(x)-1$, where $p(x)$ is $C^{\infty}$, decreasing, and $p(x)=1$ for $x \leqq 1.1, p(x)=0$ for $x \geqq 1.25$.

Let $h_{4}(x, y)=h_{3}(x, y)+q(x)$ where $q(x)$ is $C^{\infty}$, decreasing, and $q(x)=0$ for 
$x \leqq 1.5, q(2)=-1$, and then define $r(x, y)$ to be $r(x, y)=s(y) \cdot\left(h_{4}(x, y)\right)+(1-s(y))$ $\cdot(-2 x+2)$, where $s$ is a $C^{\infty}$ decreasing map, $s(y)=1$ for $y \leqq 1.5, s(y)=0$ for $y \geqq 1.75$. This function $r$ satisfies the conditions of the first sentence of the lemma, and clearly may be further modified to satisfy the conditions of the second sentence also.

Now define a homotopy

$$
F: D_{2}^{k+1} \times D_{2}^{r} \times D^{l-1} \times[0,1] \rightarrow R \times D^{l-1}
$$

by

$$
F(x, y, z, \tau)=((1-\tau) \cdot(-2|x|+2)+\tau \cdot r(|x|,|y|), z) .
$$

Then $s_{3} \circ s_{2} \circ s_{1} \circ F$ defines a homotopy of $f$ restricted to the tubular neighborhood of $h\left(D_{2}^{k+1}\right)$ in our coordinates, which is stationary on the boundary of this tubular neighborhood. Thus we may think of $s_{3} \circ s_{2} \circ s_{1} \circ F$ as defining a homotopy of $f: M \rightarrow Q$ to a new map which we denote $f_{1}: M \rightarrow Q$, since

$$
s_{3} \circ s_{2} \circ s_{1} \circ F(x, y, z, 0)=s_{3} \circ s_{2} \circ s_{1}(-2|x|+2, z)=f(x, y, z) .
$$

Note that $s_{3} \circ s_{2} \circ s_{1} \circ F(x, y, z, 1)=s_{3} \circ s_{2} \circ s_{1}(r(|x|,|y|), z)$ is differentiable in a neighborhood of the universe image of $p \in P$ and is transverse regular over $p \in P$, with the inverse image of $p$ diffeomorphic to

$$
\begin{aligned}
D^{k+1} \times S^{r-1} & =D^{k+1} \times S^{m-l-k-1} & & \text { in Case (a) } \\
& =D^{k+1} \times S^{m-l-k} & & \text { in Case (b). }
\end{aligned}
$$

Thus we end with a pair $\left(M, f_{1}\right)$, where $f_{1}: M \rightarrow Q$ is differentiable and transverse regular over $P \subset Q$ and $f_{1}^{-1}(P)$ is just the manifold $N=f^{-1}(P)$ with a surgery done on the class of $i\left(S^{k}\right)$. As noted before, the map $f_{1}$ is tangential, so we have regained our original assumptions and can proceed inductively.

Now consider the separate cases:

Case (a): It is clear from the work of Novikov [7], [12] that one can proceed inductively through a series of surgeries as above to arrive at a pair $(M, \bar{f})$, where $\bar{f}$ is differentiable and transverse to $P \subset Q$ and $\bar{f} \mid \bar{f}^{-1}(P): \bar{f}^{-1}(P) \rightarrow P$ induces isomorphisms of homology for all dimensions less than or equal to $(m-l) / 2-1$, and $\bar{f}^{-1}(P)$ simply connected. That is, surgeries as above can be used to kill the kernel of $H_{k}\left(f^{-1}(P)\right) \rightarrow H_{k}(P)$ for $k \leqq(m-l) / 2-1$. The cases $m-l \equiv 0,1,3 \bmod 4$ are distinct.

If $m-l \equiv 0 \bmod 4$, then ordinary surgery may be done to the pair $\left(\bar{f}^{-1}(P)\right.$, $\left.\bar{f} \mid \bar{f}^{-1}(P)\right)$ until homology isomorphism results in the middle dimension also. (A proof of this may be found in [7, pp. 26-28], or [12, pp. 288-290]. One must choose the homology classes in $\operatorname{ker} f_{*}$ to be killed with care.) It is further clear that these surgeries can be done ambiently as above, since the only obstruction to this is that we make a specific choice of framing of $\nu\left(i\left(S^{k}\right) \subset N\right)$ in Lemma 1.5, and the proof that surgery can be used to kill the kernel of $f_{*}$ in dimension $(m-l) / 2$ is independent of the specific framing used in performing the surgery. Hence, for $m-l \equiv 0 \bmod 4$, surgery may be completed in the middle dimension, and we end with a map 
$f^{\prime}: M \rightarrow Q$ transverse to $P \subset Q$ such that $f^{\prime} \mid f^{\prime-1}(P): f^{\prime-1}(P) \rightarrow P$ is a tangential homotopy equivalence as required in Theorem 1.

For $m-l \equiv 1 \bmod 4$ the same argument applies. Ordinary surgery may be done to $\left(\bar{f}^{-1}(P), \bar{f} \mid \bar{f}^{-1}(P)\right)$ until homology isomorphism results in dimension $(m-l-1) /$ 2 , as proven in [12, pp. 294-302]. Again one sees these surgeries can all be done ambiently in $M$ as above, since the proof is independent of the choice of frame on $\nu\left(i\left(S^{k}\right) \subset N\right)$. Thus we again end with a $\operatorname{map} f^{\prime}: M \rightarrow Q$ as required by Theorem 1 .

For $m-l \equiv 3 \bmod 4$, we must use the note following Lemma 1.5. Novikov proves that surgery can modify $\left(\bar{f}^{-1}(P), \bar{f} \mid \bar{f}^{-1}(P)\right)$ so that homology isomorphisms in dimension $(m-l-1) / 2$ result, but this proof requires use of specific framings on $\nu\left(i\left(S^{(m-l-1) / 2}\right) \subset N\right)$ in performing the surgeries. The freedom to vary a given framing by any stably trivial element of $\left.\pi_{(m-l-1) / 2}(S O(m-l+1) / 2)\right)$ is, however, all that is needed. (See [12, pp. 298-302].) Since we may use these frames in doing ambient surgery, we again end with a map $f^{\prime}: M \rightarrow Q$ as required in Theorem 1 , and Theorem 1 is proven.

Case (b). Recall in this case we have $f: M^{m+1} \rightarrow Q$ a tangential homotopy equivalence, where $M^{m+1}$ is an $h$-cobordism between $M_{1}^{m}$ and $M_{2}^{m}$, and we are doing surgery to $N=f^{-1}(P)$. Note first that since we are doing surgeries on classes represented by imbeddings $i: S^{k} \rightarrow N$ where $i\left(S^{k}\right) \cap \partial M=\varnothing$, the homotopies of $f$ we define may be assumed stationary on $\partial M$.

As in Novikov [12], [7, Proposition 4, p. 19] we get a sequence of modifications as above such that we arrive at a pair $\left(M^{m+1}, f_{1}\right)$ where $f_{1}$ is differentiable and transverse to $P$, and $f_{1 *}: H_{*}\left(f_{1}^{-1}(P)\right) \rightarrow H_{*}(P)$ is an isomorphism in dimensions less than or equal to $(m-l) / 2-1$, and $f_{1}^{-1}(P)$ simply connected. $f_{1}^{-1}(P)$ has dimension $m+1-l$, where $m-l \equiv 0 \bmod 4$, so we may refer to [7, pp. 28-35], $\left[12\right.$, p. 304] for a proof that ordinary surgery may be done to the pair $\left(f_{1}^{-1}(P)\right.$, $\left.f_{1} \mid f_{1}^{-1}(P)\right)$ until we get a pair with homology isomorphism in dimension $(m-l) / 2$ as well, and thus get a homotopy equivalence.

Once again, the only obstruction to performing these surgeries ambiently as above is the choice of framing of $\nu\left(i\left(S^{(m-l) / 2}\right) \subset f_{1}^{-1}(P)\right)$ made in Lemma 1.5. Since the proof that surgeries can be chosen to kill the kernel of $f_{1 *}: H_{(m-l) / 2}\left(f_{1}^{-1}(P)\right) \rightarrow$ $H_{(m-l) / 2}(P)$ is independent of such choices, the surgeries may all be done in $M^{m+1}$ as above.

Thus we can modify $\left(M^{m+1}, f\right)$ to a pair $\left(M^{m+1}, f^{\prime}\right)$ by a series of homotopies of $f$ so that $f^{\prime}: M \rightarrow Q$ is a tangential homotopy equivalence transverse to $P \subset Q$, $f\left|\partial M=f^{\prime}\right| \partial M$, and if $N=f^{\prime-1}(P)$, then $f^{\prime} \mid N: N \rightarrow P$ is a homotopy equivalence, so that $N$ is an $h$-cobordism between $N_{1}$ and $N_{2}$. Thus Theorem 2 is proven.

Note 1. The reader should notice that the following weaker analogue of the uniqueness theorem can be proven for the cases $m-l \equiv 1$ or $3 \bmod 4$, using the analogue of Case (b) above:

Let $P$ and $Q$ be as in Theorem 1 , with $m-l \equiv 1$ or $3 \bmod 4$. Assume $M^{m+1}$, $f: M^{m+1} \rightarrow Q, M_{i}$ and $N_{i}$ for $i=1,2$ are all as in Theorem 2, with $f \mid N_{i}: N_{i} \rightarrow P$ a 
homotopy equivalence for $i=1,2$. Then $f$ is homotopic via a homotopy which is stationary on $\partial M^{m+1}$ to a map $f^{\prime}: M^{m+1} \rightarrow Q$ such that $\pi_{1}\left(f^{\prime-1}(P)\right)=0$ and $f_{*}^{\prime}: H_{i}\left(f^{\prime-1}(P)\right) \rightarrow H_{i}(P)$ is an isomorphism for $i \leqq(m-l+1) / 2-1$. As in [12, pp. 304-306], $N_{1}$ is diffeomorphic to $N_{2} \# \Sigma^{m-l}$ for some homotopy sphere $\Sigma^{m-l}$ which bounds a parallelizable manifold.

Note 2. Before proceeding we should note that the following stronger analogue of Theorem 1 holds for manifolds with boundary.

TheOREM. Suppose $Q^{m}$ and $P^{m-l}$ and $M^{m}$ are manifolds with boundary with $P^{m-l}$ a submanifold of $Q^{m}$ so that $\partial P \subset \partial Q$, where $l \geqq 3, m-l>6, m-l \neq 14$, and $\pi_{1}(Q)=$ $\pi_{1}(\partial Q)=\pi_{1}(P)=\pi_{1}(\partial P)=0$. Suppose $f:\left(M^{m}, \partial M^{m}\right) \rightarrow\left(Q^{m}, \partial Q^{m}\right)$ is a degree +1 tangential homotopy equivalence. Then $f$ is homotopic to a map $f^{\prime}:\left(M^{m}, \partial M^{m}\right) \rightarrow$ $\left(Q^{m}, \partial Q^{m}\right)$ which is differentiable and transverse regular over $(P, \partial P) \subset(Q, \partial Q)$ and such that $f^{\prime} \mid f^{\prime-1}(P, \partial P): f^{\prime-1}(P, \partial P) \rightarrow(P, \partial P)$ is a homotopy equivalence.

The proof of this is similar in spirit to the work above. One readily shows the analogues of Lemma 1.1 and Proposition 1.1. Ambient surgery is then carried out on $f^{-1}(\partial P)$ and $f^{-1}(P)$ as above up to the middle dimension. The obstructions to middle dimensional surgery can be made to vanish since we allow our homotopies to alter $f$ on $\partial M^{m}$. The interested reader will find a discussion and proof of this, including the necessary algebra and the techniques needed to avoid middle dimension obstructions, in Wagoner [17] and Wall [18].

2. Homotopy quaternionic projective spaces. We refer the reader to the introduction for the definition of $\theta\left(Q P_{n}\right)$, which is defined for $n \geqq 2$.

LEMMA 2.1. The equivalence relation defining $\theta\left(Q P_{n}\right)$ can be described as follows: $(M, f) \sim(N, g)$ if and only if $M$ and $N$ are diffeomorphic by a map $d: M \rightarrow N$ such that $f$ is homotopic to $g \circ d$.

Proof. $(M, f) \sim(N, g)$ in $\theta\left(Q P_{n}\right)$ implies there is an $h$-cobordism $C$ and a tangential homotopy equivalence $h: C \rightarrow Q P_{n}$. Since dimension $M, N$ is greater than or equal to 5 , there is a diffeomorphism $d: M \times I \rightarrow C$ with $d(x, 0)=x$. Hence $d \mid(M \times(1))$ is a diffeomorphism $M \rightarrow N$ such that $h \circ d \mid(M \times(1))$ is homotopic to $h \circ d \mid(M \times(0))$. Since $h$ restricts to $f$ and $g, f \circ d$ is homotopic to $g \circ d$, and so $f$ is homotopic to $g \circ d$.

Conversely, if $(N, g)$ represents a class in $\theta\left(Q P_{n}\right)$ then $g \circ$ projection: $N \times I \rightarrow Q P_{n}$ is a tangential homotopy equivalence. Assume $d: M \rightarrow N$ is a diffeomorphism such that $g \circ d$ is homotopic to $f: M \rightarrow Q P_{n}$. Identify $N \times(1) \subset N \times I$ with $M$ via $d$ to make $N \times I$ an $h$-cobordism of $N$ and $M$. Then $h=g \circ$ projection restricts to $g$ on $N \times(0)$ and to a map homotopic to $f$ on $M=N \times(1)$, and may be modified in a tubular neighborhood of $M$ in $N \times I$ to restrict to $f$.

Lemma 2.2. Suppose $f: M^{4 n} \rightarrow Q P_{n}$ is a tangential homotopy equivalence and $\Sigma^{4 n}$ represents an element of $\theta_{4 n}$. Let $i: D^{4 n} \rightarrow M$ be an imbedding. Then for some 
diffeomorphism of boundaries $d: \partial D^{4 n} \rightarrow \partial\left(M-i\left(D^{\circ 4 n}\right)\right)$, the connected sum $M \# \Sigma^{4 n}$ is diffeomorphic to $\left(M-i\left(D^{\circ 4 n}\right)\right) \cup \cup_{d} D^{4 n}$. Define $\mathrm{id}^{\prime}: M \# \Sigma^{4 n} \rightarrow M$ by $\operatorname{id}^{\prime}(x)=x$ for $x \in M-i\left(D^{\circ 4 n}\right)$, and $\operatorname{id}^{\prime}(s, \tau)=i\left(i^{-1}(d(s)), \tau\right)$ for $(s, \tau) \in D^{4 n}$, where we view $D^{4 n}$ as $\left\{(s, \tau) \mid s \in S^{4 n-1}, \tau \in I\right\}$ modulo $(s, 0) \sim(r, 0)$ for any $s, r \in S^{4 n-1}$. Then $\mathrm{id}^{\prime}$ is a tangential map, and thus $f \circ \mathrm{id}^{\prime}: M \# \Sigma^{4 n} \rightarrow Q P_{n}$ is a tangential homotopy equivalence.

Proof. The existence of the required diffeomorphism $d: \partial D^{4 n} \rightarrow \partial\left(M-i\left(D^{\circ 4 n}\right)\right)$ is proven by Smale [13], so all that remains is to show id' is tangential.

$\mathrm{id}^{\prime} \mid\left(M-i\left(D^{\circ 4 n}\right)\right)$ is identity, so we may collapse $\left(M-i\left(D^{\circ 4 n}\right)\right)$ to a point to get a diagram with stable bundles

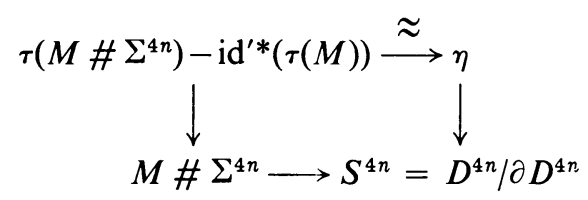

where the diagram defines the stable bundle $\eta$. We need only show $\eta$ is trivial to prove id' is tangential.

Since a stable bundle over $S^{4 n}$ is trivial if and only if its $n$th Pontrjagin class is zero, we investigate the $n$th Pontrjagin class of $\tau\left(M \# \Sigma^{4 n}\right)-\mathrm{id}^{\prime *}(\tau(M))$ which is zero if and only if the $n$th Pontrjagin class of $\eta$ is. In the Mayer-Vietoris cohomology sequence of the triad $\left(M \# \Sigma^{4 n} ; M-i\left(D^{\circ 4 n}\right), D^{4 n}\right)$ inclusion: $M-i\left(D^{\circ 4 n}\right) \rightarrow$ $M \# \Sigma^{4 n}$ induces isomorphism of cohomology for all dimensions less than $4 n-2$. Let $p_{1}, \ldots, p_{n}$ denote the Pontrjagin classes of $M$, and $p_{1}^{\prime}, \ldots, p_{n}^{\prime}$ the Pontrjagin classes of $M \# \Sigma^{4 n}$. Since $\mathrm{id}^{\prime} \mid\left(M-i\left(D^{\circ 4 n}\right)\right)$ is tangential, $p_{1}^{\prime}=\mathrm{id}^{\prime *}\left(p_{1}\right), \ldots, p_{n-1}^{\prime}$ $=\mathrm{id}^{\prime *}\left(p_{n-1}\right)$. index $\left(M \# \Sigma^{4 n}\right)=\operatorname{index}(M)$, so if $L_{n}$ is the $n$th Hirzebruch polynomial, then

$$
\begin{aligned}
L_{n}\left(p_{1}^{\prime}, \ldots, p_{n}^{\prime}\right)\left[M \# \Sigma^{4 n}\right] & =L_{n}\left(p_{1}, \ldots, p_{n}\right)[M] \\
& =L_{n}\left(\operatorname{id}^{\prime *}\left(p_{1}\right), \ldots, \mathrm{id}^{\prime *}\left(p_{n}\right)\right)\left[M \# \Sigma^{4 n}\right] \\
& =L_{n}\left(p_{1}^{\prime}, \ldots, p_{n-1}^{\prime}, \operatorname{id}^{\prime *}\left(p_{n}\right)\right)\left[M \# \Sigma^{4 n}\right]
\end{aligned}
$$

where we orient $M \# \Sigma^{4 n}$ so that id' is a degree +1 map.

If $L_{n}\left(X_{1}, \ldots, X_{n}\right)$ is the $n$th Hirzebruch polynomial, the coefficient of $X_{n}$ is nonzero. Thus we must have $p_{n}^{\prime}=\mathrm{id}^{*}\left(p_{n}\right)$ above, and hence the $n$th Pontrjagin class of $\tau\left(M \# \Sigma^{4 n}\right)-\mathrm{id}^{\prime *}(\tau(M))$ is zero and $\eta$ is trivial as needed.

Definition. $\theta_{4 n}$ acts on $\theta\left(Q P_{n}\right)$ in the following manner: $\left[\Sigma^{4 n}\right] \cdot[(M, f)] \equiv$ [ $\left.\left(M \# \Sigma^{4 n}, f \circ \mathrm{id}^{\prime}\right)\right]$. This action is well defined by Lemma 2.2 of Kervaire-Milnor [6].

Definition. Define $r: \theta\left(Q P_{n}\right) \rightarrow \theta\left(Q P_{n-1}\right)$ for all $n \geqq 3$ as follows: Given $\alpha \in \theta\left(Q P_{n}\right)$, we choose a representative element $(M, f) . Q P_{n}$ has dimension $4 n$, and codimension of $Q P_{n-1}$ in $Q P_{n}$ is $4, Q P_{n}$ is simply connected for all $n$, so by Theorem 1 and the fact that we are free within the class $\alpha$ to deform $f$ by arbitrary homotopies, we may assume $(M, f)$ is chosen with $f$ differentiable and transverse to 
$Q P_{n-1} \subset Q P_{n}$, and such that $f \mid f^{-1}\left(Q P_{n-1}\right): f^{-1}\left(Q P_{n-1}\right) \rightarrow Q P_{n-1}$ is a tangential homotopy equivalence. Define $r(\alpha)$ to be the class of $\left(f^{-1}\left(Q P_{n-1}\right), f \mid f^{-1}\left(Q P_{n-1}\right)\right)$ in $\theta\left(Q P_{n-1}\right)$. To show that the image of $\alpha$ depends only on $\alpha$, suppose $(N, g) \in \alpha$ is such that $g: N \rightarrow Q P_{n}$ is transverse to $Q P_{n-1} \subset Q P_{n}$, and $g \mid g^{-1}\left(Q P_{n-1}\right)$ : $g^{-1}\left(Q P_{n-1}\right) \rightarrow Q P_{n-1}$ is a homotopy equivalence. Let $(C, h)$ be a cobordism between $(M, f)$ and $(N, g)$ as in the definition of equivalence in $\theta\left(Q P_{n}\right)$. By Theorem 2, we may assume $h: C \rightarrow Q P_{n}$ is transverse regular over $Q P_{n-1} \subset Q P_{n}$, and that $h \mid h^{-1}\left(Q P_{n-1}\right): h^{-1}\left(Q P_{n-1}\right) \rightarrow Q P_{n-1}$ is a tangential homotopy equivalence. Then $\left(h^{-1}\left(Q P_{n-1}\right), h \mid h^{-1}\left(Q P_{n-1}\right)\right)$ is a cobordism between $\left(f^{-1}\left(Q P_{n-1}\right), f \mid f^{-1}\left(Q P_{n-1}\right)\right)$ and $\left(g^{-1}\left(Q P_{n-1}\right), g \mid g^{-1}\left(Q P_{n-1}\right)\right)$, and so these represent the same class in $\theta\left(Q P_{n-1}\right)$.

Lemma 2.3. Suppose $\left(M, f_{1}\right)$ represents $\alpha \in \theta\left(Q P_{n}\right)$, with $n \geqq 3$. Then $f_{1}$ is homotopic to $f: M \rightarrow Q P_{n}$ such that

(a) $f$ is differentiable and transverse regular over $Q P_{n-1} \subset Q P_{n}$, and

is a representative of $r(\alpha)$.

$$
\left(f^{-1}\left(Q P_{n-1}\right), f \mid f^{-1}\left(Q P_{n-1}\right)\right)
$$

(b) If we consider $Q P_{n}$ as the Thom space of $\nu\left(Q P_{n-1} \subset Q P_{n}\right)$, then $f$ defines a bundle isomorphism $\nu\left(f^{-1}\left(Q P_{n-1}\right) \subset M\right) \approx \nu\left(Q P_{n-1} \subset Q P_{n}\right)$ on a tubular neighborhood $\nu$ of $f^{-1}\left(Q P_{n-1}\right)$, and $f$ maps the complement of $\nu$ to the distinguished point in $Q P_{n}=T\left(\nu\left(Q P_{n-1} \subset Q P_{n}\right)\right)$.

Proof. See Thom [16] and the definition of $r(\alpha)$.

Lemma 2.4. For any $\alpha \in \theta\left(Q P_{n}\right)$ with $n \geqq 3$, if a representative pair $(M, f)$ for $\alpha$ is chosen as in Lemma 2.3 then the tubular neighborhood $\nu$ of $f^{-1}\left(Q P_{n-1}\right)$ in $M$ is bounded by a sphere, and $M$ is diffeomorphic to $\nu \cup_{i} D^{4 n}$ for some attaching diffeomorphism of boundaries $i: \partial D^{4 n} \rightarrow \partial \nu$.

Proof. There are disc bundle isomorphisms $\nu \approx f^{*}\left(\nu\left(Q P_{n-1} \subset Q P_{n}\right)\right) \approx f^{*}\left(\mathscr{S}^{4}\right)$, where $\mathscr{S}^{4}$ is the 4-disc bundle over $Q P_{n-1}$ described in the introduction. Hence there is a 3 -sphere bundle isomorphism $\partial \nu \approx f^{*}\left(\mathscr{S}_{0}^{3}\right)$, where $\mathscr{S}_{0}^{3}$ has total space $S^{4 n-1}$. Then naturality of the homotopy sequence of a bundle implies $\partial \nu$ has the homotopy type of $S^{4 n-1}$.

Let $D=\partial \nu \cup$ (complement of $\nu$ in $M$ ), so that $\nu \cup D=M, \nu \cap D=\partial \nu$. From the Mayer-Vietoris sequence of this decomposition of $M$ we see inclusion induces homology isomorphisms $H_{i}(v) \approx H_{i}(M)$ for $i \leqq 4 n-1$, and so $H_{i}(D)=0$ for $i \leqq 4 n-2$. By duality, $H_{4 n}(D) \approx H^{0}(D, \partial \nu)=0$ and $H_{4 n-1}(D) \approx H^{\prime}(D, \partial \nu)=0$, so $H_{i}(D)=0$ for all $i \leqq 4 n$.

Applying Van Kampen's Theorem to $M$ with subsets $\nu^{\circ}=$ interior of $\nu$, and $M-f^{-1}\left(Q P_{n-1}\right)$, we see $D$ is simply connected, since $D$ has the homotopy type of $M-f^{-1}\left(Q P_{n-1}\right)$. Hence $D$ is contractible, and $\partial D=\partial \nu$ is diffeomorphic to $S^{4 n-1}$, as needed. See [13].

Definition. Define $h: \theta\left(Q P_{n-1}\right) \rightarrow \theta_{4 n-1}$ for $n \geqq 3$ as follows. Given $\beta \in \theta\left(Q P_{n-1}\right)$ choose a representative $(P, t)$ in which $t: P \rightarrow Q P_{n-1}$ is differentiable. As in the 
proof of Lemma $2.4, t^{*}\left(\mathscr{S}_{0}^{3}\right)$ has the homotopy type of $S^{4 n-1}$. We define $h(\beta)$ to be the class in $\theta_{4 n-1}$ represented by $t^{*}\left(\mathscr{S}_{0}^{3}\right)$, and check that $h$ is now a well defined map.

If $(P, t) \sim(R, s)$ in $\theta\left(Q P_{n-1}\right)$, where both $s$ and $t$ are differentiable maps, we may choose a cobordism between them $\left(C^{\prime}, h^{\prime}\right)$ such that $h^{\prime}: C^{\prime} \rightarrow Q P_{n-1}$ is differentiable. Then $h^{\prime *}\left(\mathscr{S}_{0}^{3}\right)$ is a manifold of the homotopy type of $S^{4 n-1}$, and is thus an $h$-cobordism between $t^{*}\left(\mathscr{S}_{0}^{3}\right)$ and $s^{*}\left(\mathscr{S}_{0}^{3}\right)$.

We now proceed with the proof of Theorem 3. Given $\alpha \in \theta\left(Q P_{n}\right)$, choose a representative $(M, f)$ for $\alpha$ as in Lemma 2.3 so that $\left[\left(f^{-1}\left(Q P_{n-1}\right), f \mid f^{-1}\left(Q P_{n-1}\right)\right)\right]$ $=r(\alpha)$, and $f: M \rightarrow Q P_{n}$ is transverse to $Q P_{n-1}$. Then $\left(f \mid f^{-1}\left(Q P_{n-1}\right)\right)^{*}\left(\mathscr{S}_{0}^{3}\right)$ $=\partial\left(\nu\left(f^{-1}\left(Q P_{n-1}\right) \subset M\right)\right) \approx S^{4 n-1}$ by Lemma 2.4 , and thus image of $r$ is contained in the kernel of $h$.

Suppose $\beta \in \theta\left(Q P_{n-1}\right)$ and $h(\beta)=\left[S^{4 n-1}\right] \in \theta_{4 n-1}$. Take $(R, s) \in \beta$ with $s: R \rightarrow$ $Q P_{n-1}$ differentiable, and identify the boundary of $s^{*}\left(\mathscr{S}^{4}\right)$ with $S^{4 n-1}$. In this fashion we attach $D^{4 n}$ to $s^{*}\left(\mathscr{S}^{4}\right)$ differentiably. Let $R_{1}=s^{*}\left(\mathscr{S}^{4}\right) \cup D^{4 n}$ be the result of such an attachment. Considering $Q P_{n}$ as $\nu\left(Q P_{n-1} \subset Q P_{n}\right) \cup D^{4 n}$, or $\mathscr{S}^{4} \cup D^{4 n}$, by identifying a tubular neighborhood of $Q P_{n-1}$ with $\mathscr{S}^{4}$, we get a homotopy equivalence $s_{1}: R_{1} \rightarrow Q P_{n}$ by radial extension of the bundle map $s^{*}\left(\mathscr{S}^{4}\right) \rightarrow \mathscr{S}^{4}$. By a proof entirely similar to that of Lemma 2.2, one can show that $s_{1}$ is a tangential map, and thus that $\left(R_{1}, s_{1}\right)$ represents an element $\gamma$ of $\theta\left(Q P_{n}\right)$. Clearly $r(\gamma)=[(R, s)]$ $=\beta \in \theta\left(Q P_{n-1}\right)$, and $\beta \in$ image $(r)$. Thus we have shown image $(r)=\operatorname{kernel}(h)$.

Suppose now $\alpha, \beta \in \theta\left(Q P_{n}\right)$ are such that $r(\alpha)=r(\beta)$. Choose representative elements $(M, f)$ and $(N, g)$ for $\alpha$ and $\beta$ respectively as in Lemma 2.3. Then there is a cobordism between $\left(f^{-1}\left(Q P_{n-1}\right), f \mid f^{-1}\left(Q P_{n-1}\right)\right)$ and $\left(g^{-1}\left(Q P_{n-1}\right), g \mid g^{-1}\left(Q P_{n-1}\right)\right)$ since $r(\alpha)=r(\beta)$, and, since $f$ and $g$ are each differentiable in the respective inverse images of $Q P_{n-1}$, we may assume this cobordism is $(C, h)$ with $h: C \rightarrow Q P_{n-1}$ differentiable.

Associated with the resulting manifold $h^{*}\left(\mathscr{S}^{4}\right)$, a differentiable disc bundle, is the boundary 3-sphere bundle $h^{*}\left(\mathscr{S}_{0}^{3}\right)$. As before, naturality of the homotopy sequence of a bundle shows that $h^{*}\left(\mathscr{S}_{0}^{3}\right)$ has the homotopy type of $S^{4 n-1}$, and thus that $h^{*}\left(\mathscr{S}_{0}^{3}\right)$ is an $h$-cobordism between

$$
\left(f \mid f^{-1}\left(Q P_{n-1}\right)\right)^{*}\left(\mathscr{S}_{0}^{3}\right) \text { and }\left(g \mid g^{-1}\left(Q P_{n-1}\right)\right)^{*}\left(\mathscr{S}_{0}^{3}\right) \text {. }
$$

Since $\left(f^{-1}\left(Q P_{n-1}\right), f \mid f^{-1}\left(Q P_{n-1}\right)\right) \in r(\alpha)$, and image $(r)=\operatorname{kernel}(h), h^{*}\left(\mathscr{S}_{0}^{3}\right)$ is thus diffeomorphic to $S^{4 n-1} \times I$.

Attach $D^{4 n} \times I$ to $h^{*}\left(\mathscr{S}^{4}\right)$ by a diffeomorphism $S^{4 n-1} \times I \approx \partial h^{*}\left(\mathscr{S}^{4}\right)$, thus creating a manifold $C^{\prime}$, and a homotopy equivalence $h^{\prime}: C^{\prime} \rightarrow Q P_{n}$, the radial extension of the natural bundle map. Then $C^{\prime}$ is an $h$-cobordism between its boundary components, which are respectively $M \# \Sigma^{4 n}$ and $M \# \Sigma^{\prime 4 n}$, where $\Sigma^{4 n}$ and $\Sigma^{\prime 4 n}$ are homotopy $4 n$-spheres. We now check that $h^{\prime}: C^{\prime} \rightarrow Q P_{n}$ is tangential, and thus that $\left(C^{\prime}, h^{\prime}\right)$ is a cobordism between $\left[\Sigma^{4 n}\right] \cdot \alpha$ and $\left[\Sigma^{\prime 4 n}\right] \cdot \beta$ in $\theta\left(Q P_{n}\right)$.

The proof that $h^{\prime}$ is tangential is as in Lemma 2.2. If $i: h^{*}\left(\mathscr{S}^{4}\right) \rightarrow C^{\prime}$ is inclusion, then $i^{*}\left(\tau\left(C^{\prime}\right)\right) \approx i^{*} h^{*}\left(\tau\left(Q P_{n}\right)\right)$ and hence the first $n-1$ Pontrjagin classes of $\tau\left(C^{\prime}\right)$ 
and $h^{\prime *}\left(\tau\left(Q P_{n}\right)\right)$ are the same. Since $\tau\left(C^{\prime}\right)$ and $h^{\prime *}\left(\tau\left(Q P_{n}\right)\right)$ are stably isomorphic over $h^{*}\left(\mathscr{S}^{4}\right) \subset C^{\prime}$, we may collapse this subspace to a line, and by analogy with Lemma 2.2 define a bundle $\eta$ over $S^{4 n} \times I$. One can then show $\eta \mid S^{4 n} \times(0)$ is stably trivial by computing its $n$th Pontrjagin class, and thus prove that $h^{\prime}: C^{\prime} \rightarrow Q P_{n}$ is tangential. Then $\left[\Sigma^{4 n}\right] \cdot \alpha$ and $\left[\Sigma^{\prime 4 n}\right] \cdot \beta$ represent the same class in $\theta\left(Q P_{n}\right)$, and $\alpha$ and $\beta$ differ by an action of $\theta_{4 n}$ in $\theta\left(Q P_{n}\right)$.

Conversely, choosing representatives as in Lemma 2.3, it is clear that if $\alpha$ and $\beta$ differ by some action of $\theta_{4 n}$, then $r(\alpha)=r(\beta)$. This completes the proof of Theorem 3 .

Construction. Theorem 3 gives us an inductive procedure whereby we can construct representatives of all elements of $\theta\left(Q P_{n}\right)$ from elements of $\theta\left(Q P_{n-1}\right)$ for $n \geqq 3$.

Given any representative $(M, f)$ of $\alpha \in \theta\left(Q P_{n}\right)$, we may deform $f$ by a homotopy and assume it is in the form prescribed by Lemma 2.3. Then $r(\alpha)$ is represented by $\left(f^{-1}\left(Q P_{n-1}\right), f \mid f^{-1}\left(Q P_{n-1}\right)\right)$, and $\left(f \mid f^{-1}\left(Q P_{n-1}\right)\right)^{*}\left(\mathscr{S}_{0}^{3}\right)$ is diffeomorphic to $S^{4 n-1}$. Attach $D^{4 n}$ to $\left(f \mid f^{-1}\left(Q P_{n-1}\right)\right)^{*}\left(\mathscr{S}^{4}\right)$ by a diffeomorphism of boundaries, thus creating a new manifold $M^{\prime}$ and a homotopy equivalence $f^{\prime}: M^{\prime} \rightarrow Q P_{n}$, which we have seen in the proof of Theorem 3 is tangential. Then $\left(M^{\prime}, f^{\prime}\right)$ represents an element of $\theta\left(Q P_{n}\right)$, and $r\left(\left[\left(M^{\prime}, f^{\prime}\right)\right]\right)=r(\alpha)$, and so by Theorem $3[(M, f)]$ and $\left[\left(M^{\prime}, f^{\prime}\right)\right]$ differ by some action of $\theta_{4 n}$ in $\theta\left(Q P_{n}\right)$. Thus we see all tangential homotopy $Q P_{n}$ 's arise from tangential $Q P_{n-1}$ 's by the operation of pulling back the bundle $\mathscr{S}^{4}$, and capping the result with a disc $D^{4 n}$.

3. $\theta\left(Q P_{2}\right)$. The existence of the inductive procedure for constructing tangential homotopy quaternionic projective spaces given in the last section leads one to investigate the starting point, $\theta\left(Q P_{2}\right)$.

Suppose $(M, f)$ represents an element of $\theta\left(Q P_{2}\right)$. Let $i: S^{4} \rightarrow M^{8}$ be an imbedding representing a generator of $\pi_{4}\left(M^{8}\right) \approx \pi_{4}\left(Q P_{2}\right) \approx Z$.

Lemma 3.1. Let $v$ denote a tubular neighborhood of $i\left(S^{4}\right)$ in $M$. Then $D=M$ interior( $v)$ is diffeomorphic to the 8-disc, $D^{8}$. Consequently, the 3-sphere bundle which bounds $v\left(i\left(S^{4}\right) \subset M\right)$ has total space diffeomorphic to $S^{7}$.

Proof. Since $M$ is simply connected and $D$ is a deformation retract of $M-i\left(S^{4}\right)$, $D$ is also simply connected. For $q<8, H^{q}(M) \approx H^{q}\left(i\left(S^{4}\right)\right)$, so $H_{j}(D) \approx H^{8-j}\left(M, i\left(S^{4}\right)\right)$ $=0$ for $j \neq 0$. Hence $D$ is contractible, and $D \approx D^{8}$ by the $h$-cobordism theorem.

We now investigate $\nu\left(i\left(S^{4}\right) \subset M\right)=\nu$. Since $f: M \rightarrow Q P_{2}$ is tangential, $\nu \oplus \tau\left(S^{4}\right)$ $\approx i^{*}(\tau(M)) \approx i^{*} f^{*}\left(\nu\left(Q P_{1} \subset Q P_{2}\right) \oplus \tau\left(S^{4}\right)\right)$, where the isomorphisms indicated are stable isomorphisms, and thus $\nu$ is stably isomorphic to $i^{*} f^{*}\left(\nu\left(Q P_{1} \subset Q P_{2}\right)\right)$.

Represent 3-sphere bundles over $S^{4}$ by elements of $\pi_{3}(S O(4))$ as in Steenrod $[14, \S \S 18-22]$, using the isomorphism described by Milnor [9], $\pi_{3}(S O(4)) \approx Z \oplus Z$ under the correspondence $(h, j) \Leftrightarrow f_{h j}$, where $f_{h j}: S^{3} \rightarrow S O(4)$ is defined by $f_{h j}(u) \cdot v=u^{h} \cdot v \cdot u^{j}$ for $v \in R^{4}$. (Quaternion multiplication is indicated on the right.) It is know that under this isomorphism $\nu\left(Q P_{1} \subset Q P_{2}\right)$ corresponds to $(1,0) \in Z \oplus Z$. See [9]. 
Lemma 3.2. If $\mathscr{S}_{h j}$ is the 3-sphere bundle over $S^{4}$ corresponding to $(h, j)$ in this representation, then the first Pontrjagin class of $\mathscr{S}_{h j}$ is $p_{1}\left(\mathscr{S}_{h j}\right)= \pm 2(h-j) i$, where $i \in H^{4}\left(S^{4}\right)$ is a generator.

Proof. See Milnor [9].

LEMMA 3.3. $\nu=\nu\left(i\left(S^{4}\right) \subset M\right)$ is represented by an element of the form

$$
(n+1, n) \in Z \oplus Z \text {. }
$$

Proof. $p_{1}\left(\tau\left(S^{4}\right)\right)=0$, so by Lemma $3.2 \tau\left(S^{4}\right)$ is represented by an element of $Z \oplus Z$ of the form $(s, s)$. Kernel $\pi_{3}\left(S O(4) \rightarrow \pi_{3}(S O)\right)=Z$, and since the element corresponding to $\tau\left(S^{4}\right)$ is in this kernel, the kernel is contained in the diagonal of $Z \oplus Z$. Thus $\nu$ is represented by $(1,0)+(n, n)=(n+1, n)$ for some $n \in Z$ since $\nu\left(Q P_{1} \subset Q P_{2}\right)$ is represented by $(1,0)$ and is stably isomorphic to $\nu$.

LeMmA 3.4. $\nu=v\left(i\left(S^{4}\right) \subset M\right) \approx \nu\left(Q P_{1} \subset Q P_{2}\right)$.

Proof. We will show that among those 3-sphere bundles over $S^{4}$ with representatives of the form $(n+1, n)$ in $\pi_{3}(S O(4)) \approx Z \oplus Z$, only the one corresponding to $(1,0)$ has a total space of the homotopy type of $S^{7}$.

Let $T$ be the total space of some 4-disc bundle over $S^{4}$ with associated 3-sphere bundle denoted by $\partial . \pi_{1}(\partial)=\pi_{2}(\partial)=0$, then, and the first possibly nonvanishing homology group of $\partial$ is $H_{3}(\partial)$. Consider the cohomology exact sequence

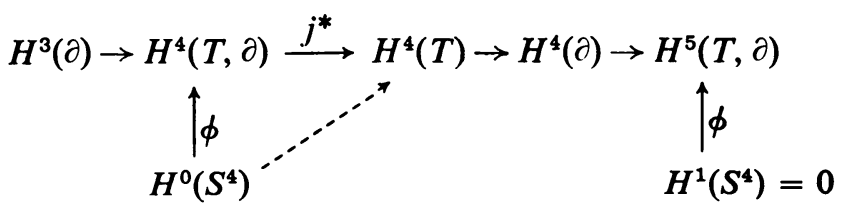

where the maps denoted by $\phi$ are the Thom isomorphisms. The image of a generator of $H^{0}\left(S^{4}\right)$ in $H^{4}(T)$ under $j^{*} \circ \phi$ is a generator if and only if $H^{4}(\partial)=0$, and it is known that the Euler class of the 4-disc bundle of which $T$ is the total space is the image of a generator $H^{0}\left(S^{4}\right)$ under $j^{*} \circ \phi$.

The process of taking Euler classes of corresponding bundles gives a homomorphism $\pi_{3}(S O(4)) \rightarrow H^{4}\left(S^{4}\right)$ (see [8]), and the Euler class of $\tau\left(S^{4}\right)$ is twice a generator of $H^{4}\left(S^{4}\right)$, so in the diagonal set $\{(n, n) \mid n \in Z\}$ of $Z \times Z$, all bundles represented have nonzero Euler classes except for $(0,0)$. The Euler class of the bundle corresponding to $(1,0)$ is a generator of $H^{4}\left(S^{4}\right)$ since this bundle is just $\partial \nu\left(Q P_{1} \subset Q P_{2}\right)$ and has total space $S^{7}$. Thus $(1,0)$ is the only element of $Z \oplus Z$ of the form $(n+1, n)$ whose corresponding bundle has Euler class a generator of $H^{4}\left(S^{4}\right)$, and $(1,0)$ is the only element of the form $(n+1, n)$ representing a 3-sphere bundle whose total space has the homotopy type of $S^{7}$. Since the total space of $\nu$ is $S^{7}$, we see $\nu \approx \nu\left(Q P_{1} \subset Q P_{2}\right)$. 
So, any manifold $M^{8}$ of the tangential homotopy type of $Q P_{2}$ is of the form $M=T \cup D^{8}$, where $T$ is the total space of the differentiable disc bundle $\nu\left(Q P_{1} \subset Q P_{2}\right)$, and $D^{8}$ is attached to $T$ by a diffeomorphism of boundaries. Thus we have proven:

Proposition 3.1. Any 8 manifold without boundary of the tangential homotopy type of $Q P_{2}$ is diffeomorphic to a connected sum $Q P_{2} \# \Sigma^{8}$, where $\Sigma^{8}$ is a homotopy 8-sphere. Thus, there are at most two distinct such manifolds, and these are combinatorially equivalent [11].

To finish the computation of $\theta\left(Q P_{2}\right)$, we investigate the homotopy classes of maps $f: M \rightarrow Q P_{2}$ which contain tangential homotopy equivalences. If $f: M \rightarrow Q P_{2}$ and $g: M \rightarrow Q P_{2}$ are two such equivalences, and if $f$ and $g$ are not homotopic, then $f \circ g^{-1}: Q P_{2} \rightarrow Q P_{2}$ is a tangential homotopy equivalence which is not homotopic to identity.

The remainder of this section is devoted to a proof that there is only one homotopy class of maps from $Q P_{2}$ to $Q P_{2}$ which contains a tangential homotopy equivalence. This will finish the proof of Theorem 4.

Let $f: Q P_{2} \rightarrow Q P_{2}$ be a tangential homotopy equivalence. It is known [3] that $p_{1}\left(Q P_{2}\right)=1$ st Pontrjagin class of $Q P_{2}$ is twice a generator in $H^{4}\left(Q P_{2}\right) \approx Z$, and thus tangentiality of $f$ implies $f_{*}: \pi_{4}\left(Q P_{2}\right) \rightarrow \pi_{4}\left(Q P_{2}\right)$ is identity. So, in the standard C-W complex structure of $Q P_{2}$ we may assume $f$ is the identity map on $S^{4}=4$ skeleton of $Q P_{2}$, and that $h_{\tau}: S^{4} \rightarrow S^{4}$, the identity map for all $\tau \in I$, is a homotopy between $f$ and identity on the 7-skeleton of $Q P_{2}$, which is the same as the 4-skeleton.

The partial homotopy of $f$ with identity given by $h_{\tau}$ gives rise to an obstruction cohomology class $\delta^{8}\left(f\right.$, identity, $\left.h_{\tau}\right) \in H^{8}\left(Q P_{2}, \pi_{8}\left(Q P_{2}\right)\right) \approx Z_{2}$, since the homotopy sequence of the bundle $S^{3} \rightarrow S^{11} \rightarrow Q P_{2}$ shows $\pi_{8}\left(Q P_{2}\right) \approx \pi_{7}\left(S^{3}\right) \approx Z_{2}$. If this obstruction class is zero then the partial homotopy $h_{\tau}$ may be extended over $Q P_{2}$ to give a homotopy of $f$ with identity. Moreover, if $g$ is another such map, and $\delta^{8}\left(f\right.$, id, $\left.h_{\tau}\right) \neq 0, \delta^{8}\left(g\right.$, id,$\left.h_{\tau}\right) \neq 0$, then $f$ and $g$ are homotopic by the additivity of obstruction cochains. Thus, we may finish the proof of the theorem by constructing a map $t: Q P_{2} \rightarrow Q P_{2}$ which is identity on the 4-skeleton of $Q P_{2}$ and which is homotopic to identity: $Q P_{2} \rightarrow Q P_{2}$, but whose obstruction class $\delta^{8}\left(t, \mathrm{id}, h_{\tau}\right)$ is not zero. (The homotopy of $t$ to identity cannot then be stationary on the 4-skeleton of $Q P_{2}$.)

We view $Q P_{2}$ as the Thom space of the 4-disc bundle $\mathscr{S}^{4}$ over $S^{4} \approx Q P_{1}$, and represent $\mathscr{S}^{4}$ as follows. $\mathscr{S}^{4}$ can be thought of as $\{(s, v) \mid s$ a quaternionic line in $Q^{2}, v$ a point in $\left.s\right\}$. Define a map from $\left\{\left.(a, b, q)|a, b, q \in Q ;| a\right|^{2}+|b|^{2}=1,|q| \leqq 1\right\}$ to $\mathscr{S}^{4}$ taking $(a, b, q)$ to (the line containing $\left.(a, b), q \cdot(a, b)\right)$. If $(a, b, q)$ and $\left(a^{\prime}, b^{\prime}, q^{\prime}\right)$ have the same image under this map then $(a, b)=s \cdot\left(a^{\prime}, b^{\prime}\right)$ for some $s \in Q,|s|=1$, and $q \cdot s \cdot\left(a^{\prime}, b^{\prime}\right)=q^{\prime} \cdot\left(a^{\prime}, b^{\prime}\right)$ so that $q=q^{\prime} \cdot s^{-1}$. Thus we can represent $\mathscr{S}^{4}$ over $S^{4}$ as $\left\{\left.(a, b, q)|a, b, q \in Q| a\right|^{2}+,|b|^{2}=1,|q| \leqq 1\right\}$ modulo the equivalence relation $(a, b, q) \sim\left(a^{\prime}, b^{\prime}, q^{\prime}\right)$ if and only if $a=s \cdot a^{\prime}, b=s \cdot b^{\prime}$ and $q=q^{\prime} \cdot s^{-1}$ for some $s \in Q$. $\mathscr{S}_{0}^{3}$ is given in this description by the set of equivalence classes which have a 
representative with third coordinate of length one. We view $S^{4}$ as $Q \cup\{\infty\}$, and identify $Q P_{1}=\left\{[(a, b, q)] \in \mathscr{S}^{4} \mid q=0\right\}$ with $S^{4}$ under the correspondence $[(a, b, 0)]$ $\Leftrightarrow a^{-1} b$, noting that if $(a, b, 0)=\left(q^{\prime} \cdot c, q^{\prime} \cdot d, 0\right)$ then $a^{-1} b=c^{-1} q^{\prime-1} q^{\prime} d=c^{-1} d$.

Let $\eta: S^{4} \rightarrow S^{3}$ be the essential map in $\pi_{4}\left(S^{3}\right) \approx Z_{2}$, where we think of $S^{3}$ as the unit length quaternions. Then, with the above identifications we define $t: Q P_{2} \rightarrow Q P_{2}$ by $t([(a, b, q)])=\left[\left(a, b, \eta\left(a^{-1} b\right) \cdot q\right)\right]$. One checks easily that this map $\mathscr{S}^{4} \rightarrow \mathscr{S}^{4}$ is well defined on equivalence classes, and maps $\mathscr{S}_{0}^{3}$ to $\mathscr{S}_{0}^{3}$ and so does in fact give a map $Q P_{2} \rightarrow Q P_{2}$. We wish to show that the obstruction to this map being homotopic to identity: $Q P_{2} \rightarrow Q P_{2}$ via a homotopy which is stationary on the 4-skeleton of $Q P_{2}$ is the nontrivial element in $\pi_{8}\left(Q P_{2}\right)$.

Identify $D^{8}$ with $\left\{\left.(a, b, c)|a, b \in Q, c \in R| a\right|^{2}+,|b|^{2}=1,0 \leqq c \leqq 1\right\}$ modulo the relation $(a, b, 0) \sim(c, d, 0)$ for any $a, b, c, d$. Then define $p: D^{8} \rightarrow \mathscr{S}^{4} / \mathscr{S}_{0}^{3}=Q P_{2}$ by $p([(a, b, c)])=\left[\left(a, b, 1-c^{2}\right)\right]$. Note that on the boundary of $D^{8}$ the map $p$ is just the Hopf map $S^{7} \rightarrow S^{4}$.

The obstruction class we wish to compute in $\pi_{8}\left(Q P_{2}\right)$ is readily seen to be represented by the following map: View $S^{8}$ as two copies of $D^{8}$ with boundaries identified. On one hemisphere we define our map to be $p: D^{8} \rightarrow Q P_{2}$ as described above. On the other hemisphere we define our map to be $t \circ p: D^{8} \rightarrow Q P_{2}$ as above. Since $t$ is identity on $S^{4}=Q P_{1} \subset Q P_{2}$ and $p$ maps $\partial D^{8}$ to this $S^{4}$, this gives a continuous well-defined map $S^{8} \rightarrow Q P_{2}$ which is the Hopf map on the "equator" of $S^{8}$.

To show this map represents the nonzero class in $\pi_{8}\left(Q P_{2}\right) \approx Z_{2}$, we will show the pull back of $\mathscr{S}^{4}$ by this map is a nontrivial bundle over $S^{8}$. Analogous to the above description of $\mathscr{S}^{4}$ over $S^{4}$, one may describe $\mathscr{S}^{4}$ over $Q P_{2}$ as

$$
\left\{\left.(a, b, c, q)|a, b, c, q \in Q,| a\right|^{2}+|b|^{2}+|c|^{2}=1,|q| \leqq 1\right\}
$$

modulo the equivalence relation $(a, b, c, q) \sim\left(a^{\prime}, b^{\prime}, c^{\prime}, q^{\prime}\right)$ if and only if for some $s \in Q, a=s \cdot a^{\prime}, b=s \cdot b^{\prime}, c=s \cdot c^{\prime}$, and $q=q^{\prime} \cdot s^{-1}$. The trivial 4-disc bundle over $D^{8}$ can be descirbed as $\left\{\left.(a, b, c, q)|a, b, q \in Q| a\right|^{2}+,|b|^{2}=1,|q| \leqq 1 ; c \in R, 0 \leqq c \leqq 1\right\}$ modulo the relation $(a, b, 0, q) \sim(c, d, 0, q)$ for any $a, b, c, d \in Q$. We may now describe a bundle map from this bundle to $\mathscr{S}^{4}$ over $Q P_{2}$ by $p^{\prime}([(a, b, c, q)])=$ $\left[\left(c \cdot a, c \cdot b, 1-c^{2}, q\right)\right]$, which is easily seen to be well defined on equivalence classes. This bundle map lies over the base space map $p: D^{8} \rightarrow Q P_{2}$, as may be seen by comparing the two descriptions of $Q P_{2}$ which are used: the Thom space of the bundle $\mathscr{S}^{4}$ over $Q P_{1}$ and the zero section of $\mathscr{S}^{4}$ over $Q P_{2}$.

A second map from the trivial 4-disc bundle over $D^{8}$ to $\mathscr{S}^{4}$ over $Q P_{2}$ is given by

$$
\begin{aligned}
(t \circ p)^{\prime}([(a, b, c, q)]) & =\left[\left(c \cdot a, c \cdot b, \eta\left(a^{-1} b\right)\left(1-c^{2}\right), q \cdot \eta\left(a^{-1} b\right)^{-1}\right)\right] \\
& =\left[\left(\eta\left(a^{-1} b\right)^{-1} \cdot c \cdot a, \eta\left(a^{-1} b\right)^{-1} \cdot c \cdot b,\left(1-c^{2}\right), q\right)\right] .
\end{aligned}
$$

Note that this map can be thought of as the composition of $p^{\prime}$ and the bundle map over $t: Q P_{2} \rightarrow Q P_{2}$ which takes " $q$ " over $[(a, b, c)]$ to " $q$ " over $t([(a, b, c)])$, and is thus a continuous bundle map over $t \circ p$. 
Looking at our representation of $\mathscr{S}^{4}$ over $Q P_{2}$ restricted to $S^{4} \subset Q P_{2}$, we see that the inverse image of the point $[(a, b, 0, q)]$ under $p^{\prime}$ is the set

$$
\left\{\left[\left(q^{\prime} a, q^{\prime} b, 1, q q^{\prime-1}\right)\right]\left|q^{\prime} \in Q,\right| q^{\prime} \mid=1\right\},
$$

and the inverse image of this same point under the map $(t \circ p)^{\prime}$ is the set

$$
\left\{\left[\left(q^{\prime} a, q^{\prime} b, 1, q q^{\prime-1} \eta\left(a^{-1} b\right)\right)\right]\left|q^{\prime} \in Q,\right| q^{\prime} \mid=1\right\} .
$$

Thus, if we take two copies of the trivial 4-disc bundle over $D^{8}$ and identify the point represented by $[(a, b, 1, q)]$ in one with the point represented by

$$
\left[\left(a, b, 1, q \cdot \eta\left(a^{-1} b\right)\right)\right]
$$

in the other, we get a bundle $\rho$ over $S^{8}=D^{8} \cup D^{8}$, and the maps $p^{\prime}$ and $(t \circ p)^{\prime}$ give a bundle map from this bundle to $\mathscr{S}^{4}$ over $Q P_{2}$ which lies over $p$ on one hemisphere, and over $t \circ p$ on the other. That is, there is a bundle map from $\rho$ to $\mathscr{S}^{4}$ which lies over the element of $\pi_{8}\left(Q P_{2}\right)$ we wish to compute.

The bundle $\rho$ has coordinate transition map $S^{7} \rightarrow S O(4)$ given by a composition $S^{7} \rightarrow S^{4} \rightarrow S^{3} \rightarrow S O(4)$ which takes $(a, b)$ to $f_{(a, b)}$ where $f_{(a, b)}(q)=q \cdot \eta\left(a^{-1} b\right)$ with quaternion multiplication indicated on the right. This map in $\pi_{7}(S O(4))$ is nontrivial (Steenrod $[14, \S 22]$ ), and thus $\rho$ is a nontrivial bundle, and the map $S^{8} \rightarrow Q P_{2}$ is not nullhomotopic. Thus we have proven that the obstruction to finding a homotopy of $t: Q P_{2} \rightarrow Q P_{2}$ to identity which is stationary on $S^{4} \subset Q P_{2}$ is nonzero.

The proof of Theorem 4 will now be completed by showing that $t: Q P_{2} \rightarrow Q P_{2}$ is in fact homotopic to identity, and thus that the class of identity is the only homotopy class of maps $Q P_{2} \rightarrow Q P_{2}$ which contains a tangential homotopy equivalence. Recall that $t$ comes from a bundle map of $\mathscr{S}^{4}$ over $S^{4}=Q P_{1}$ to itself which lies over identity: $S^{4} \rightarrow S^{4}$. The principal bundle associated with $\mathscr{S}^{4}$ is $\mathscr{S}_{0}^{3}$, with fiber and group $S^{3}=$ the group of unit length quaternions. Since $\mathscr{S}_{0}^{3}$ over $S^{4}$ has total space $S^{7}, \mathscr{S}^{4}$ is a universal bundle for disc bundles with group $S^{3}$ over complexes of dimension less than or equal to six [14]. Thus, there is a homotopy of $t$ to the identity, $T: \mathscr{S}^{4} \times I \rightarrow \mathscr{S}^{4}$, a bundle map which is $t$ on $\mathscr{S}^{4} \times(0)$ and identity on $\mathscr{S}^{4} \times(1)$. Since $T$ maps $\mathscr{S}_{0}^{3} \times I$ to $\mathscr{S}_{0}^{3}$, this induces a map $Q P_{2} \times I \rightarrow Q P_{2}$ which is the required homotopy of $t$ to identity.

4. Combinatorial equivalence. In this section the results of the preceding two sections are applied to investigate $\theta\left(Q P_{n}\right)$ for all $n \geqq 2$.

Lemma 4.1. Suppose $(M, f)$ and $(N, g)$ represent the same element in $\theta\left(Q P_{n}\right)$ for some $n \geqq 2$, and that $g: N \rightarrow Q P_{n}$ is homotopic to a map $h: N \rightarrow Q P_{n}$ which is induced by a combinatorial equivalence. Then $f: M \rightarrow Q P_{n}$ is also homotopic to a map induced by a combinatorial equivalence.

Proof. Suppose $\tau_{1}: K_{1} \rightarrow N, \tau_{2}: K_{2} \rightarrow Q P_{n}$, and $c: K_{1} \rightarrow K_{2}$ give a combinatorial equivalence which induces $h$. Recall from Lemma 2.1 that there is a diffeomorphism 
$d: N \rightarrow M$ such that $g$ is homotopic to $f \circ d$. Then $d \circ \tau_{1}: K_{1} \rightarrow M$ is a $C^{\infty}$ triangulation of $M$. Let $h^{\prime}=h \circ d^{-1}: M \rightarrow Q P_{n}$. Then $h^{\prime}$ is induced by the combinatorial equivalence given by $d \circ \tau_{1}, \tau_{2}$, and $c$. Further, $f \circ d$ homotopic to $g$ implies $f$ homotopic to $h^{\prime}$.

Lemma 4.2. Let $\tau_{1}: K_{1} \rightarrow M$ and $\tau_{2}: K_{2} \rightarrow M$ be $C^{\infty}$ triangulations of the manifold $M$. Then for any Riemannian metric on $M$ and any $\varepsilon>0$, there is a combinatorial equivalence $c: K_{1} \rightarrow K_{2}$ such that the induced map $h=\tau_{2} \circ c \circ \tau_{1}^{-1}$ is an $\varepsilon$-approximation to identity.

Proof. Given $\varepsilon>0$, choose $\varepsilon / 2$ approximations $\tau_{1}^{\prime}: K_{1}^{\prime} \rightarrow M$ and $\tau_{2}^{\prime}: K_{2}^{\prime} \rightarrow M$ to $\tau_{1}$ and $\tau_{2}$ respectively where $K_{i}^{\prime}$ is a subdivision of $K_{i}$ for $i=1,2$ as in [10, p. 101] such that for some combinatorial equivalence $c^{\prime}: K_{1}^{\prime} \rightarrow K_{2}^{\prime}, \tau_{2}^{\prime} \circ c^{\prime} \circ \tau_{1}^{\prime-1}: M \rightarrow M$ is identity. Since $K_{1}^{\prime}$ and $K_{2}^{\prime}$ are subdivisions of $K_{1}$ and $K_{2}, c^{\prime}$ induces a combinatorial equivalence $c: K_{1} \rightarrow K_{2}$. Then $h=\tau_{2} \circ c \circ \tau_{1}^{-1}$ is an $\varepsilon$-approximation to identity, and by definition is induced by $c$.

Proposition 4.1. If $(M, f)$ is any representative of an element of $\theta\left(Q P_{2}\right)$, then $f: M \rightarrow Q P_{2}$ is homotopic to a map induced by a combinatorial equivalence.

Proof. By Lemma 4.1, we need only prove the proposition for one representative in each equivalence class in $\theta\left(Q P_{2}\right)$. The proposition is obvious for $\left(Q P_{2}\right.$, identity), so, by Theorem 4, we need only work with $\left(Q P_{2} \# \Sigma^{8}, \mathrm{id}^{\prime}\right)$ where id' is defined as in Lemma 2.2 by a map $\phi:\left(Q P_{2}-i\left(D^{\circ 8}\right)\right) \cup_{d} D^{8} \rightarrow\left(Q P_{2}-i\left(D^{\circ 8}\right)\right) \cup_{i} D^{8}$ such that $\phi(x)=x$ for $x \in Q P_{2}-i\left(D^{\circ 8}\right)$, and $\phi(s, \tau)=\left(d^{-1}(s), \tau\right)$ for $(s, \tau) \in D^{8}$, for $d: S^{7} \rightarrow S^{7}$ a representative of the nonzero element of $\Gamma_{8}$.

We now refer to $\left[11\right.$, p. 546] where for each $n$ a $C^{\infty}$-triangulation $g_{n}: K^{n} \rightarrow D^{n}$ is constructed having the property that if $h: S^{n-1} \rightarrow S^{n-1}$ is any map which induces a combinatorial equivalence of $\partial\left|K^{n}\right|$ with itself then the radial extension of $h$ to a map $h_{r}: D^{n} \rightarrow D^{n}$ induces a combinatorial equivalence of $K^{n}$ with itself.

Proceed as in [11]. Consider the triangulations of $\partial\left(Q P_{2}-i\left(D^{\circ}\right)\right)$ given by restriction to the boundary of $i \circ g_{8}: K^{8} \rightarrow Q P_{2}-i\left(D^{\circ 8}\right)$, and of $d \circ i \circ g_{8}: K^{8} \rightarrow$ $Q P_{2}-i\left(D^{\circ 8}\right)$. Each of these $C^{\infty}$-triangulations has an extension over all of $Q P_{2}-i\left(D^{\circ 8}\right)$ as in [10, p. 101], and, by Lemma 4.2, we may choose a combinatorial equivalence $c$ of these triangulations such that the map $h$ induced by this equivalence is homotopic to identity via a homotopy which maps $\partial\left(Q P_{2}-i\left(D^{\circ 8}\right)\right) \times I$ to $\partial\left(Q P_{2}-i\left(D^{\circ 8}\right)\right)$. Restricted to $\partial\left(Q P_{2}-i\left(D^{\circ 8}\right)\right), h$ gives a combinatorial equivalence $c|\partial| K_{1} \mid$. Thus the radial extension of $h$ to a map $h^{\prime}:\left(Q P_{2}-i\left(D^{\circ 8}\right)\right) \cup_{d} D^{8} \rightarrow$ $\left(Q P_{2}-i\left(D^{\circ}\right)\right) \cup_{i} D^{8}$ gives a combinatorial equivalence, and extending the homotopy of $h$ to identity radially gives a homotopy of $h^{\prime}$ to id' as needed.

Proposition 4.2. Suppose $f: M \rightarrow Q P_{n}$ for $n \geqq 2$ is differentiable so that there is a natural differentiable structure on the pullback $f^{*}\left(\mathscr{S}^{4}\right)$, and that $p: f^{*}\left(\mathscr{S}^{4}\right) \rightarrow \mathscr{S}^{4}$ is a map induced by a combinatorial equivalence. Let $i: D^{4 n+4} \rightarrow Q P_{n+1}$ be an imbedding such that $i\left(D^{4 n+4}\right) \cap\left(Q P_{n} \subset Q P_{n+1}\right)=\varnothing$, and identify $Q P_{n+1}$ with 
$\mathscr{S}^{4} \cup_{i} D^{4 n+4}$. If $d: S^{4 n+3} \rightarrow \partial f^{*}\left(\mathscr{S}^{4}\right)$ is a diffeomorphism, and we form the manifold $f^{*}\left(\mathscr{S}^{4}\right) \cup \cup_{d} D^{4 n+4}$ and extend $p$ to $p_{d}: f^{*}\left(\mathscr{S}^{4}\right) \cup{ }_{d} D^{4 n+4} \rightarrow \mathscr{S}^{4} \cup{ }_{i} D^{4 n+4}$ by $p_{d}(x)=$ $p(x)$ for $x \in f^{*}\left(\mathscr{S}^{4}\right), p_{d}(s, \tau)=\left(i^{-1}(p(d(s))), \tau\right)$ for $(s, \tau) \in D^{4 n+4}$, then $p_{d}$ is homotopic to a map $p_{d}^{\prime}: f^{*}\left(\mathscr{S}^{4}\right) \cup \cup_{d} D^{4 n+4} \rightarrow \mathscr{S}^{4} \cup_{i} D^{4 n+4}$ which is induced by a combinatorial equivalence.

Proof. Again we will use Munkres' triangulation $g_{4 n+4}: K^{4 n+4} \rightarrow D^{4 n+4}$ and proceed as in [11]. $d \circ g_{4 n+4}$ and $p \circ d \circ g_{4 n+4}$ are respectively $C^{\infty}$-triangulations of $\partial f^{*}\left(\mathscr{S}^{4}\right)$ and $\partial \mathscr{S}^{4}$. These extend to triangulations $\tau_{3}: K_{3} \rightarrow f^{*}\left(\mathscr{S}^{4}\right)$ and $\tau_{4}: K_{4} \rightarrow \mathscr{S}^{4}$ respectively, as in [10, p. 101]. By Lemma 4.2 , there are combinatorial equivalences $c_{1}$ and $c_{2}$ such that in the following diagram $i_{1}$ and $i_{2}$ are approximations to the identity maps close enough so that $i_{1}$ and $i_{2}$ are each homotopic to identity via homotopies which always carry $\partial f^{*}\left(\mathscr{S}^{4}\right)$ to $\partial f^{*}\left(\mathscr{S}^{4}\right)$ and $\partial \mathscr{S}^{4}$ to $\partial \mathscr{S}^{4}$. In the diagram, $c$ is a combinatorial equivalence, and $p=\tau_{2} \circ c \circ \tau_{1}^{-1}$, where $\tau_{1}$ and $\tau_{2}$ are $C^{\infty}$-triangulations.

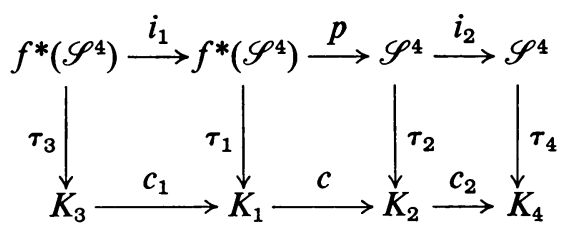

Restricted to $\partial f^{*}\left(\mathscr{S}^{4}\right)$, the map $i_{2} \circ p \circ i_{1}$ induces the equivalence $c_{2} \circ c \circ c_{1}$ on $\partial K^{4 n+4}$, and thus the radial extension of $i_{2} \circ p \circ i_{1}$ induces a combinatorial equivalence $K^{4 n+4} \rightarrow K^{4 n+4}$. So, triangulating $D^{4 n+4}$ by $g_{4 n+4}$, we get

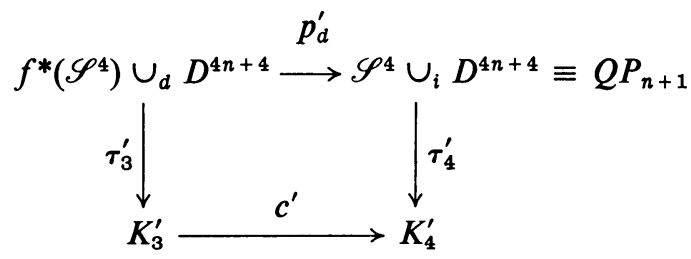

where $c^{\prime}$ is a combinatorial equivalence and $\tau_{3}^{\prime}, \tau_{4}^{\prime}$ are $C^{\infty}$-triangulations and $p_{d}^{\prime}$ is the radial extension of $i_{2} \circ p \circ i_{1}$. But $p_{d}^{\prime}$ is homotopic to the radial extension of $p$ via radial extensions of the homotopies of $i_{1}$ and $i_{2}$ to identity maps, and the proposition is proven.

Lemma 4.3. Suppose $f: \sigma^{p} \rightarrow R^{n}$ is a map of a p-simplex into $R^{n}$ such that for some subdivision of $\partial \sigma, f \mid \partial \sigma$ is differentiable on each simplex of $\partial \sigma$. Then $f$ is homotopic to a map $f^{\prime}: \sigma^{p} \rightarrow R^{n}$ such that $f^{\prime}|\partial \sigma=f| \partial \sigma$, and for some subdivision of $\sigma, f^{\prime}$ is differentiable on each simplex in $\sigma$.

Proof. Assume $\sigma^{p} \subset R^{p}$ such that $b_{\sigma}=$ barycenter of $\sigma$ is at the origin of $R^{p}$. Let $l: R^{p} \rightarrow R^{p}$ be defined by $l(x)=x / 2$, and let $N=\sigma-l(\sigma)$. Subdivide $\sigma$ and its faces so that $f \mid \partial \sigma$ is differentiable on each simplex of $\partial \sigma$ and triangulate $l(\partial \sigma)$ by the composition of this triangulation of $\partial \sigma$ and $l$. Sets of the form $\{x \in N \mid \tau \cdot x \in \gamma$ 
for some $\tau, 1 \leqq \tau \leqq 2\}$ as $\gamma$ ranges over the simplices of $\partial \sigma$, the simplices of $\partial \sigma$, and the images of the simplices of $\partial \sigma$ under $l$ give a cellular decomposition of $N[10$, p. 70].

As in [10], this cell complex may be divided into a simplicial complex without subdividing any of the cells of $\partial N=\partial \sigma \cup l(\partial \sigma)$, since these cells are already simplices. Define $f^{\prime}: \partial N \rightarrow R^{n}$ by $f^{\prime}|\partial \sigma=f| \partial \sigma$, and $f^{\prime} \mid l(\partial \sigma)$ is that map which is linear on each simplex of $l(\partial \sigma)$, and such that $f$ and $f^{\prime}$ agree on the vertices of $l(\partial \sigma)$. Given $x \in N$, let $\tau(x)$ be the real number between 1 and 2 such that $\tau(x) \cdot x \in \partial \sigma$. Then define $f^{\prime}$ on the interior of $N$ by

$$
f^{\prime}(x)=(2-\tau(x)) \cdot f(\tau(x) \cdot x)+(\tau(x)-1) \cdot f^{\prime}(\tau(x) \cdot x / 2) .
$$

Then $f^{\prime}$ is differentiable on each simplex of the triangulation of $N$, and $f^{\prime}$ is linear on each simplex in $\partial(l(\sigma))$. We triangulate $l(\sigma)$ by taking the join of $b_{\sigma}$ with the simplices in $\partial(l(\sigma))$, and extend $f^{\prime}$ linearly over the simplices in $l(\sigma)$, letting $f^{\prime}\left(b_{\sigma}\right)$ $=f\left(b_{\sigma}\right)$, to get the required map $f^{\prime}: \sigma \rightarrow R^{n}$.

LeMma 4.4. Let $\sigma^{p}$ be a simplex in Euclidean space. Then any $C^{\infty}$-triangulation of $\partial(\sigma) \times D^{k}$ has a subdivision which can be extended to a $C^{\infty}$-triangulation of $\sigma \times D^{k}$.

Proof. The proof is in two parts; first extend a subdivision to a triangulation of $\partial\left(\sigma \times D^{k}\right)$, and then extend a subdivision of this to the needed triangulation of $\sigma \times D^{k}$.

Suppose $\tau: K \rightarrow \partial(\sigma) \times D^{k}$ is a $C^{\infty}$-triangulation. $\partial\left(\sigma \times D^{k}\right)=\partial(\sigma) \times D^{k} \cup \sigma \times \partial D^{k}$, where the intersection $\partial \sigma \times \partial D^{k}$ already has a $C^{\infty}$-triangulation then. Subdivide $K$ so that in this triangulation of $\partial \sigma \times \partial D^{k}$ every simplex $\gamma$ of the subdivided complex which is mapped by $\tau$ into $\partial \sigma \times \partial D^{k}$ is mapped into a face of $\sigma$ by (projection $\circ \tau$ ): $K \rightarrow \partial \sigma$.

Let $r: \sigma^{p} \rightarrow D^{p}$ be a map as in $\left[11\right.$, p. 546] which is a $C^{\infty}$-triangulation of $D^{p}$ if we take the first barycentric subdivision of $\sigma$, and define a map $(\partial \sigma) \times\left(\partial D^{k}\right) \rightarrow$ $S^{p-1} \times S^{k-1}$ by $(x, v) \rightarrow(r(x), v)$. This induces a $C^{\infty}$-triangulation of $S^{p-1} \times S^{k-1}$ which can be extended [10] to a $C^{\infty}$-triangulation of $D^{p} \times S^{k-1}$. We further subdivide this extension so that it pulls back to a $C^{\infty}$-triangulation of $\partial\left(\sigma \times D^{k}\right)$ which extends a subdivision of $K$. Denote this triangulation of $\partial\left(\sigma \times D^{k}\right)$ by

$$
\tau^{\prime}: K^{\prime} \rightarrow \partial\left(\sigma \times D^{k}\right) \text {. }
$$

Let $b_{\sigma}$ denote the barycenter of $\sigma$, and 0 the center of $D^{k}$. For some integer $s \geqq 2$, the distance from $b_{\sigma}$ to $\partial \sigma$ is greater than $2 / s$. For $(x, y) \in \sigma \times D^{k}-\left(b_{\sigma}, 0\right)$, let $p(x, y)$ denote the unique point of $\partial\left(\sigma \times D^{k}\right)$ which lies on the ray from $\left(b_{\sigma}, 0\right)$ through $(x, y)$, and $d(x, y)$ the distance from $(x, y)$ to $p(x, y)$. Let

$$
N=\left\{(x, y) \in \sigma \times D^{k} \mid d(x, y) \leqq 1 / s\right\},
$$

and define $p_{1}: N \rightarrow \partial\left(\sigma \times D^{k}\right) \times[0,1 / s]$ by $p_{1}(x, y)=(p(x, y), d(x, y))$. Then $p_{1}$ is a homeomorphism, and

(1) for $\gamma$ any face of $\sigma, p_{1} \mid p_{1}^{-1}\left(\gamma \times D^{k}\right)$ is a $C^{\infty}$-map of rank equal to $\operatorname{dim} \gamma+k$,

(2) $p_{1} \mid p_{1}^{-1}\left(\sigma \times \partial D^{k}\right)$ is a $C^{\infty}$-map of rank $p+k-1$. 
Proceed as in [10, p. 102]. Subdivide $K^{\prime}$ to a complex $K^{2}$ such that the image of each simplex of $K^{2}$ lies in a subset of $\partial\left(\sigma \times D^{k}\right)$ of the form $\gamma \times D^{k}$ for $\gamma$ a face of $\sigma$, or in $\sigma \times \partial D^{k}$. Then triangulate $\partial\left(\sigma \times D^{k}\right) \times[0,1 / s]$ by first triangulating $\partial\left(\sigma \times D^{k}\right) \times I$ as in [10, p. 102] and then mapping $\partial\left(\sigma \times D^{k}\right) \times I \rightarrow \partial\left(\sigma \times D^{k}\right) \times[0,1 / s]$ by $(x, \tau) \rightarrow$ $(x, \tau / s)$. Then $p_{1}^{-1}$ composed with this triangulation of $\partial\left(\sigma \times D^{k}\right) \times[0,1 / s]$ gives a $C^{\infty}$-triangulation of $N$ which extends $\tau^{\prime}: K^{2} \rightarrow \partial\left(\sigma \times D^{k}\right)$. As in [10], we fit this triangulation of $N$ together with a $C^{\infty}$-triangulation of interior $\left(\sigma \times D^{k}\right)$ to get the needed triangulation of $\sigma \times D^{k}$.

Proposition 4.3. Assume $f: M \rightarrow Q P_{n}$ is homotopic to a map $h$ induced by a combinatorial equivalence. Then $f$ is homotopic to a differentiable map $f_{1}: M \rightarrow Q P_{n}$ such that the natural map $\bar{f}_{1}: f_{1}^{*}\left(\mathscr{S}^{4}\right) \rightarrow \mathscr{S}^{4}$ is homotopic to a map induced by a combinatorial equivalence, and the homotopy may be taken so that it always maps $\partial\left(f_{1}^{*}\left(\mathscr{S}^{4}\right)\right)$ to $\partial\left(\mathscr{S}^{4}\right)$.

Proof. We may choose trivialization of $\mathscr{S}^{4}$ over a finite number, say $m$, of coordinate neighborhoods in $Q P_{n}$ such that

(1) the trivializations are given by maps $\tau_{i}: D^{\circ 4 n} \times D^{4} \rightarrow \mathscr{S}^{4}$ for $1 \leqq i \leqq m$, where $D^{\circ 4 n}=\left\{x \in D^{4 n}|| x \mid<1\right\}$, and the induced base space maps $\tau_{i} \mid D^{\circ 4} \times(0)$ are diffeomorphisms onto their images which we denote by $0_{i} \subset Q P_{n}$.

(2) $Q P_{n} \subset \bigcup_{i=1}^{m} \tau_{i}\left(D_{1-\varepsilon}^{4 n} \times(0)\right)$ for some $\varepsilon>0$ where $D_{1-\varepsilon}^{4 n}=\left\{x \in D^{4 n}|| x \mid \leqq 1-\varepsilon\right\}$.

(3) the coordinate bundle transition maps in $\mathscr{S}^{4}$ induced by the $\tau_{i}, g_{i j}: 0_{i} \cap 0_{j} \rightarrow$ $S O(4)$ are $C^{\infty}$-maps for all $i, j, 1 \leqq i, j \leqq m$.

Assume $\tau_{1}: K_{1} \rightarrow M$ and $\tau_{2}: K_{2} \rightarrow Q P_{n}$ are $C^{\infty}$-triangulations, and $c: K_{1} \rightarrow K_{2}$ is a combinatorial equivalence such that $h=\tau_{2} \circ c \circ \tau_{1}^{-1}$. Since $f$ is homotopic to $h$, $f$ is homotopic to a differentiable map $f_{1}$ which is an $\varepsilon / 5$ approximation to $h$ in some Riemannian metric on $Q P_{n}$ for which the trivialization maps $\tau_{i}$ all induce distance increasing maps on base spaces. Assume $K_{1}$ and $K_{2}$ are subdivided so that $c: K_{1} \rightarrow K_{2}$ is a simplicial isomorphism, and thus $h \circ \tau_{1}: K_{1} \rightarrow Q P_{n}$ is a $C^{\infty}$ triangulation.

For any $i, 1 \leqq i \leqq m, h^{-1}\left(0_{i}\right) \cap f_{1}^{-1}\left(0_{i}\right)$ contains $h^{-1}\left(\tau_{i}\left(D_{1-\varepsilon / 2}^{4 n} \times(0)\right)\right.$, and hence sets of the form $h^{-1}\left(0_{i}\right) \cap f_{1}^{-1}\left(0_{i}\right)$ form an open cover of $M$. We assume $K_{1}$ has been subdivided so that for each simplex $\sigma$ of $K_{1}$ there is an $i$ such that

$$
\tau_{1}(\sigma) \subset h^{-1}\left(0_{i}\right) \cap f_{1}^{-1}\left(0_{i}\right) .
$$

We pull the trivialization we have selected of $\mathscr{S}^{4}$ over $0_{i}$ back to a trivialization of $h^{*}\left(\mathscr{S}^{4}\right)$ over $h^{-1}\left(0_{i}\right)$ and of $f_{1}^{*}\left(\mathscr{S}^{4}\right)$ over $f_{1}^{-1}\left(0_{i}\right)$ for each $i$. Given any $4 n$-simplex $\sigma$ of $K_{1}$, trivialize $h^{*}\left(\mathscr{S}^{4}\right) \mid \tau_{1}(\sigma)$ and $f_{1}^{*}\left(\mathscr{S}^{4}\right) \mid \tau_{1}(\sigma)$ by fixing some choice of $i$ such that $\tau_{1}(\sigma) \subset f_{1}^{-1}\left(0_{i}\right) \cap h^{-1}\left(0_{i}\right)$ and using the corresponding trivialization over $\tau_{1}(\sigma)$. This induces a trivialization of $h^{*}\left(\mathscr{S}^{4}\right) \mid \tau_{1}(\gamma)$ and of $f_{1}^{*}\left(\mathscr{S}^{4}\right) \mid \tau_{1}(\gamma)$ for any face $\gamma$ of $\sigma$, and in this fashion fixes trivializations of $h^{*}\left(\mathscr{S}^{4}\right)$ and $f_{1}^{*}\left(\mathscr{S}^{4}\right)$ over each of the simplices of the triangulation of $M$ given by $\tau_{1}: K_{1} \rightarrow M$.

Let $H: M \times I \rightarrow Q P_{n}$ be a homotopy with $H|M \times(0)=h, H| M \times(1)=f_{1}$, and 
identify $H^{*}\left(\mathscr{S}^{4}\right) \mid M \times(0)$ with $h^{*}\left(\mathscr{S}^{4}\right)$ and $H^{*}\left(\mathscr{S}^{4}\right) \mid M \times(1)$ with $f_{1}^{*}\left(\mathscr{S}^{4}\right)$. Then a bundle isomorphism $H^{*}\left(\mathscr{S}^{4}\right) \approx h^{*}\left(\mathscr{S}^{4}\right) \times I$ gives us a bundle isomorphism

$$
b_{1}: h^{*}\left(\mathscr{S}^{4}\right) \rightarrow f_{1}^{*}\left(\mathscr{S}^{4}\right)
$$

over identity: $M \rightarrow M$ such that if $\bar{h}: h^{*}\left(\mathscr{S}^{4}\right) \rightarrow \mathscr{S}^{4}$ and $\bar{f}_{1}: f_{1}^{*}\left(\mathscr{S}^{4}\right) \rightarrow \mathscr{S}^{4}$ are the natural maps then $\bar{h} b_{1}^{-1}$ is homotopic as a bundle map to $\bar{f}_{1}$.

Given an arbitrary simplex $\sigma$ of $K_{1}$, it is possible that $\sigma$ is a face of several $4 n$ simplices, and thus that $h^{*}\left(\mathscr{S}^{4}\right)$ and $f_{1}^{*}\left(\mathscr{S}^{4}\right)$ are each trivialized above in several ways over $\tau_{1}(\sigma)$. If specific trivializations of $h^{*}\left(\mathscr{S}^{4}\right)$ and $f_{1}^{*}\left(\mathscr{S}^{4}\right)$ are fixed over $\tau_{1}(\sigma), b_{1}$ induces a map $\sigma \times D^{4} \rightarrow \sigma \times D^{4}$ which is represented by a map $\sigma \rightarrow S O(4)$. If trivializations pulled back from the trivialization $\tau_{i}$ of $\mathscr{S}^{4}$ over $0_{i}$ are used, we will denote this induced map by $b_{\sigma}^{i}: \sigma \rightarrow S O(4)$. If trivializations pulled back from $\tau_{j}$ over $0_{j}$ are used, we get $b_{\sigma}^{j}$ where $b_{\sigma}^{j}=\left(g_{i j} \circ f_{1} \circ \tau_{1}\right) \cdot b_{\sigma}^{i} \cdot\left(g_{j i} \circ h \circ \tau_{1}\right)$ where the dots indicate composition of elements in $S O(4)$. Thus if $b_{\sigma}^{i}$ is a $C^{\infty}$-map, then $b_{\sigma}^{j}$ is also $C^{\infty}$.

Subdivide $K_{1}$ so that for all simplices $\gamma \in K_{1}$ and all $i, b_{\gamma}^{i}(\gamma)$ is contained in a coordinate neighborhood $U_{\gamma}^{i}$ in the differentiable structure of $S O(4)$. For each $\gamma \in K_{1}$, and all $i$, choose a diffeomorphism $d_{\gamma}^{i}: U_{\gamma}^{i} \rightarrow R^{6}$. We smooth the map $b_{1}$ as follows:

Suppose $p \geqq 1$ and $b_{1}$ is such that all of the induced maps $b_{\sigma}^{i}$ are $C^{\infty}$ for all simplices $\sigma$ of $K_{1}$ of dimension less than or equal to $p-1$. Let $\gamma$ be a $p$-simplex of $K_{1}$. Choose some $i, 1 \leqq i \leqq m$ such that $\gamma$ is a $p$-face of a $4 n$-simplex $\sigma$ where $\tau_{1}(\sigma) \subset h^{-1}\left(0_{i}\right) \cap f_{1}^{-1}\left(0_{i}\right)$, and thus fix a choice of one of our trivializations of $h^{*}\left(\mathscr{S}^{4}\right)$ and $f_{1}^{*}\left(\mathscr{S}^{4}\right)$ over $\tau_{1}(\gamma)$. For any face $\alpha$ of $\gamma, b_{\gamma}^{i} \mid \alpha: \alpha \rightarrow S O(4)$ is $C^{\infty}$ by assumption, and so $d_{\gamma}^{i} \circ b_{\gamma}^{i}: \alpha \rightarrow R^{6}$ is $C^{\infty}$ for all faces $\alpha$ of $\gamma$. By Lemma 4.3, we may choose a map $c_{\gamma}^{i}: \gamma \rightarrow R^{6}$ homotopic to $d_{\gamma}^{i} \circ b_{\gamma}^{i}$ such that $c_{\gamma}^{i}\left|\partial \gamma=d_{\gamma}^{i} \circ b_{\gamma}^{i}\right| \partial \gamma$ and $c_{\gamma}^{i}$ is a $C^{\infty}$-map on each simplex of some subdivision of $\gamma$. Then $d_{\gamma}^{i-1} \circ c_{\gamma}^{1}$ : $\gamma \rightarrow S O(4)$ is equal to $b_{y}^{i}$ on $\partial \gamma$ and differentiable on each simplex of some subdivision of $\gamma$. Thus $b_{1}$ can be deformed by a bundle homotopy which is stationary over the image under $\tau_{1}$ of the $p$-skeleton of $K_{1}$ minus $\gamma$ so that the induced map of the resulting bundle map over $\tau_{1}(\gamma)$ in the trivializations of $h^{*}\left(\mathscr{S}^{4}\right)$ and $f_{1}^{*}\left(\mathscr{S}^{4}\right)$ over $\tau_{1}(\gamma)$ corresponding to $i$ is $d_{\gamma}^{i-1} \circ c_{\gamma}^{i}$. Do this for all $p$-simplices $\gamma$ of $K_{1}$ to get a bundle map which we again denote by $b_{1}$ such that the induced maps $b_{\gamma}^{i}$ are all $C^{\infty}$-maps on some subdivision of $\gamma$ for all $\gamma$ of dimension less than or equal to $p$. Since all maps $b_{\sigma}^{i}$ are in fact $C^{\infty}$ for $\sigma$ of dimension 0 , we see by induction that we may subdivide $K_{1}$ to a complex $K$ and deform $b_{1}$ by a series of bundle homotopies to a map $b$ such that all induced maps $b_{\sigma}^{i}: \sigma \rightarrow S O(4)$ are $C^{\infty}$ for all $\sigma \in K, 1 \leqq i \leqq m$.

Since $K$ is a subdivision of $K_{1}$, each $4 n$-simplex of $K$ is contained in a unique $4 n$-simplex of $K_{1}$, and so our choices of trivializations of $h^{*}\left(\mathscr{S}^{4}\right)$ and $f_{1}^{*}\left(\mathscr{S}^{4}\right)$ over the simplices $\tau_{1}(\sigma)$ for $\sigma \in K$, induce choices of trivializations over the simplices $\tau_{1}(\gamma)$ for $\gamma \in K$. Let $\gamma$ be a simplex of $K$. We will need the following facts:

(1) If $\gamma$ is a face of two different $4 n$-simplices $\sigma_{1}$ and $\sigma_{2}$ of $K$, the bundle 
$h^{*}\left(\mathscr{S}^{4}\right) \mid \boldsymbol{\tau}_{1}(\gamma)$ is trivialized above in two possibly distinct ways. The map induced by this $\gamma \times D^{4} \rightarrow \gamma \times D^{4}$ is a diffeomorphism.

Proof. This map is a diffeomorphism if and only if the map induced $\gamma \rightarrow S O(4)$ is a $C^{\infty}$-map. If coordinates over $\tau_{1}\left(\sigma_{1}\right)$ come from the trivializing map $\tau_{i}$ and coordinates over $\tau_{1}\left(\sigma_{2}\right)$ come from $\tau_{j}$, the map $\gamma \rightarrow S O(4)$ is $g_{i j} \circ h \circ \tau_{1}: \gamma \rightarrow S O(4)$. Since $h \circ \tau_{1}: K \rightarrow Q P_{n}$ is a $C^{\infty}$-triangulation, we may extend $g_{i j} \circ h \circ \tau_{1}$ to a $C^{\infty}$-map on a neighborhood $N(\gamma)$ of $\gamma$ in Euclidean space, and the map $\gamma \times D^{4} \rightarrow$ $\gamma \times D^{4}$ is a diffeomorphism.

(2) Using one of our choices of trivialization of $h^{*}\left(\mathscr{S}^{4}\right) \mid \tau_{1}(\gamma)$ we map $\gamma \times D^{4}$ to $h^{*}\left(\mathscr{S}^{4}\right) \mid \tau_{1}(\gamma)$. Then the composition

$$
\gamma \times D^{4} \longrightarrow h^{*}\left(\mathscr{S}^{4}\right) \mid \tau_{1}(\gamma) \stackrel{\hbar}{\longrightarrow} \mathscr{S}^{4}
$$

is a diffeomorphism onto its image where $\bar{h}$ is the natural map.

Proof. Since $h \circ \tau_{1} \mid \gamma: \gamma \rightarrow Q P_{n}$ is a $C^{\infty}$-map of rank equal to the dimension of $\gamma$, and we have chosen $\tau_{i}$ so that the map $0_{i} \times D^{4} \rightarrow \mathscr{S}^{4}$ is a diffeomorphism onto its image, this is clear.

(3) Suppose for some simplex $\sigma$ and one of our choices of trivialization of $h^{*}\left(\mathscr{S}^{4}\right) \mid \tau_{1}(\gamma)$, we have a map $\sigma \rightarrow h^{*}\left(\mathscr{S}^{4}\right) \mid \tau_{1}(\gamma)$ such that the composition $\sigma \rightarrow \gamma \times D^{4}$ is a $C^{\infty}$-map of rank equal to the dimension of $\sigma$. Then, by (1) above, for any of the other choices of trivialization of $h^{*}\left(\mathscr{S}^{4}\right) \mid \tau_{1}(\gamma)$ fixed above, the composition $\sigma \rightarrow \gamma \times D^{4}$ is a $C^{\infty}$-map of rank equal to $\operatorname{dim} \sigma$.

We will call a triangulation of $h^{*}\left(\mathscr{S}^{4}\right)$ differentiable if the image of each simplex $\sigma$ of the triangulation is contained in $h^{*}\left(\mathscr{S}^{4}\right) \mid \tau_{1}(\gamma)$ for some $\gamma \in K$, and the triangulation is such that the induced map $\sigma \rightarrow \gamma \times D^{4}$ for at least one, and thus by (3) all, of our choices of trivialization of $h^{*}\left(\mathscr{S}^{4}\right) \mid \tau_{1}(\gamma)$ is a $C^{\infty}$-map of rank equal to $\operatorname{dim} \sigma$. We now construct such a triangulation of $h^{*}\left(\mathscr{S}^{4}\right)$ inductively.

Assume $h^{*}\left(\mathscr{S}^{4}\right) \mid \tau_{1}\left(K^{p}\right)$, where $K^{p}=$ the $p$-skeleton of $K$, is differentiably triangulated in this sense by $\tau_{p}^{\prime}: K^{\prime p} \rightarrow h^{*}\left(\mathscr{S}^{4}\right) \mid \tau_{1}\left(K^{p}\right)$. (Note then $\operatorname{dim} K^{\prime p}=p+4$.) Let $\sigma^{p+1}$ be any $(p+1)$-simplex of $K$, and choose one of the trivializations we have fixed for $h^{*}\left(\mathscr{S}^{4}\right) \mid \tau_{1}(\sigma)$. Then $h^{*}\left(\mathscr{S}^{4}\right) \mid \tau_{1}(\partial \sigma)$ is already triangulated by the restriction of $\tau_{p}^{\prime}$ to a subcomplex $K_{\sigma}^{\prime p}$ of $K^{\prime p}$. Using the trivialization map $h^{*}\left(\mathscr{S}^{4}\right) \mid \tau_{1}(\sigma) \approx \sigma \times D^{4}$, we get a $C^{\infty}$-triangulation $K_{\sigma}^{\prime p} \rightarrow(\partial \sigma) \times D^{4}$. By Lemma 4.4 for $k=4$, there is a differentiable triangulation of $\sigma \times D^{4}$ which extends a subdivision of $\tau_{p}^{\prime}: K_{\sigma}^{\prime p} \rightarrow$ $(\partial \sigma) \times D^{4}$. Thus there is a complex $\bar{K}_{\sigma}^{p}$ which contains a subdivision of $K_{\sigma}^{\prime p}$ and a map $\bar{\tau}_{p}: \bar{K}_{\sigma}^{p} \rightarrow h^{*}\left(\mathscr{S}^{4}\right) \mid \tau_{1}(\sigma)$ which extends $\tau_{p}^{\prime}$ and which is a differentiable triangulation of $h^{*}\left(\mathscr{S}^{4}\right) \mid \tau_{1}(\sigma)$ in the above defined sense. We adjoint $\bar{K}_{\sigma}^{p}$ to a subdivision of $K^{\prime p}$ by identifying subdivisions of $K_{\sigma}^{\prime p}$ so that the resulting complex gives a differentiable triangulation of $h^{*}\left(\mathscr{S}^{4}\right) \mid \tau_{1}\left(K^{p} \cup \sigma\right)$.

Proceeding in this fashion for each $(p+1)$-simplex $\sigma$ of $K$, we obtain a complex $K^{p+1}$ which contains a subdivision of $K^{\prime p}$, and a map

$$
\tau_{p+1}^{\prime}: K^{\prime p+1} \rightarrow h^{*}\left(\mathscr{S}^{4}\right) \mid \tau_{1}\left(K^{p+1}\right)
$$


which extends $\tau_{p}^{\prime}$ and which is a differentiable triangulation of $h^{*}\left(\mathscr{S}^{4}\right) \mid \tau_{1}\left(K^{p+1}\right)$. Since $h^{*}\left(\mathscr{S}^{4}\right) \mid \tau_{1}\left(K^{0}\right)$ may easily be triangulated differentiably, we see by induction that there is a differentiable triangulation $\tau^{\prime}: K^{\prime} \rightarrow h^{*}\left(\mathscr{S}^{4}\right)$.

For any simplex $\sigma^{\prime}$ of $K^{\prime}$, then, there is some $\sigma \in K$ such that $\tau^{\prime}\left(\sigma^{\prime}\right) \subset h^{*}\left(\mathscr{S}^{4}\right) \mid \tau_{1}(\sigma)$, and for any of the trivializations of $h^{*}\left(\mathscr{S}^{4}\right) \mid \tau_{1}(\sigma)$, we have fixed, the induced map $\sigma^{\prime} \rightarrow \sigma \times D^{4}$ is a $C^{\infty}$-map of rank equal to $\operatorname{dim} \sigma^{\prime}$. By property (2) above of the trivialization maps, $\bar{h} \circ \tau^{\prime} \mid \sigma^{\prime}$ is a $C^{\infty}$-map of rank equal to $\operatorname{dim} \sigma^{\prime}$ also, and hence $\bar{h} \circ \tau^{\prime}: K^{\prime} \rightarrow \mathscr{S}^{4}$ is a $C^{\infty}$-triangulation of $\mathscr{S}^{4}$.

Again, for $\sigma^{\prime}$ a simplex of $K^{\prime}$, we choose $\sigma \in K$ such that $\tau^{\prime}\left(\sigma^{\prime}\right) \subset h^{*}\left(\mathscr{S}^{4}\right) \mid \tau_{1}(\sigma)$. If trivializations in $h^{*}\left(\mathscr{S}^{4}\right)$ and $f_{1}^{*}\left(\mathscr{S}^{4}\right)$ induced from $\tau_{i}$ in $\mathscr{S}^{4}$ over $0_{i}$ are used, then the induced maps $\sigma^{\prime} \rightarrow h^{*}\left(\mathscr{S}^{4}\right) \rightarrow \sigma \times D^{4}$ and thus

$$
\sigma^{\prime} \rightarrow \sigma \times D^{4} \rightarrow \sigma \times D^{4} \rightarrow f_{1}^{*}\left(\mathscr{S}^{4}\right)
$$

are $C^{\infty}$ and of rank equal to $\operatorname{dim} \sigma^{\prime}$, where the map $\sigma \times D^{4} \rightarrow \sigma \times D^{4}$ is given by $(x, v) \rightarrow\left(x, b_{\sigma}^{i}(x) \cdot v\right)$ and $\sigma \times D^{4} \rightarrow f_{1}^{*}\left(\mathscr{S}^{4}\right)$ is the trivialization map. But this is just $b \circ \tau^{\prime} \mid \sigma^{\prime}$, so $b \circ \tau^{\prime}: K^{\prime} \rightarrow f_{1}^{*}\left(\mathscr{S}^{4}\right)$ is a $C^{\infty}$-triangulation.

Thus $\bar{h} \circ \tau^{\prime}: K^{\prime} \rightarrow \mathscr{S}^{4}$ and $b \circ \tau^{\prime}: K^{\prime} \rightarrow f_{1}^{*}\left(\mathscr{S}^{4}\right)$ are $C^{\infty}$-triangulations, and $\bar{h} b^{-1}: f_{1}^{*}\left(\mathscr{S}^{4}\right) \rightarrow \mathscr{S}^{4}$ is a map induced by a combinatorial equivalence, identity: $K^{\prime} \rightarrow K^{\prime} . \bar{h} b^{-1}$ is homotopic to $\bar{h} b_{1}^{-1}$ which is homotopic to $\bar{f}_{1}$, so the proposition is proven.

The proof of Theorem 5 can now be given: Proposition 4.1 is just Theorem 5 for the case $n=2$. We proceed by induction on $n$.

Assume Theorem 5 is known true for all $m$ satisfying $2 \leqq m \leqq r-1$, and that $(M, f)$ represents an element of $\theta\left(Q P_{r}\right)$. By the results of $\S 2,(M, f) \sim(N, g)$ in $\theta\left(Q P_{r}\right)$ where $g: N \rightarrow Q P_{r}$ is differentiable and transverse regular over $Q P_{r-1} \subset Q P_{r}$ and $\left[\left(g^{-1}\left(Q P_{r-1}\right), g \mid g^{-1}\left(Q P_{r-1}\right)\right)\right]=r([(M, f)])$. Then

$$
(S, \tau) \equiv\left(g^{-1}\left(Q P_{r-1}\right), g \mid g^{-1}\left(Q P_{r-1}\right)\right)
$$

satisfies the theorem, and by Proposition 4.3, $\tau$ is homotopic to a differentiable map $\tau_{1}: S \rightarrow Q P_{r-1}$ such that $\bar{\tau}_{1}: \tau_{1}^{*}\left(\mathscr{S}^{4}\right) \rightarrow \mathscr{S}^{4}$ is homotopic as a bundle map to a map induced by a combinatorial equivalence. $[(S, \tau)]=\left[\left(S, \tau_{1}\right)\right] \in$ image $(r)$ implies $\partial\left(\tau_{1}^{*}\left(\mathscr{S}^{4}\right)\right)=\tau_{1}^{*}\left(\mathscr{S}_{0}^{3}\right)$ is diffeomorphic to $S^{4 r-1}$, and if we choose a diffeomorphism $d$ and attach $D^{4 r}$ to $\tau_{1}^{*}\left(\mathscr{S}^{4}\right)$ using $d$ then $\bar{\tau}_{1}$ extends radially to a tangential homotopy equivalence $\tilde{\tau}: \tau_{1}^{*}\left(\mathscr{S}^{4}\right) \cup_{d} D^{4 r} \rightarrow Q P_{r}$. By Proposition 4.2, $\tilde{\tau}$ is homotopic to a map induced by a combinatorial equivalence.

Since $r\left(\left[\left(\tau_{1}^{*}\left(\mathscr{S}^{4}\right) \cup \cup_{d} D^{4 r}, \tilde{\tau}\right)\right]\right)=r([(N, g)])$, there is some choice of attaching diffeomorphism $d$ such that $[(N, g)]=\left[\left(\tau_{1}^{*}\left(\mathscr{S}^{4}\right) \cup_{d} D^{4 r}, \tilde{\tau}\right)\right]$ in $\theta\left(Q P_{r}\right)$. Since $(M, f) \sim$ $(N, g)$, Lemma 4.1 shows $f: M \rightarrow Q P_{r}$ is homotopic to a map induced by a combinatorial equivalence. Hence Theorem 5 is proven for all integers $m$ satisfying $2 \leqq m \leqq r$, and by induction the theorem is proven.

Note. It seems likely that the more sophisticated techniques of Sullivan [15] could be applied to prove Theorem 5 . 


\section{BIBLIOGRAPHY}

1. A. Haefliger, Plongements différentiables de variétés dans variétés, Comment. Math. Helv. 36 (1961), 820-823.

2. - Knotted (4k-1)-spheres in 6k-space, Ann. of Math. (2) 75 (1962), 452-466.

3. F. Hirzebruch, Uber die quaternionalen projectiven Räume, S.-B. Math.-Nat. Kl. Bayer. Akad. Wiss. 1953, 301-312.

4. Wu-Chung, Hsiang, A note on free differentiable actions of $S^{1}$ and $S^{3}$ on homotopy spheres, Ann. of Math. 83 (1966), 266-272.

5. Wu-Chung Hsiang, J. Levine and R. Szczarba, On the normal bundle of a homotopy sphere embedded in Euclidean space, Topology 3 (1965), 173-181.

6. M. Kervaire and J. Milnor, Groups of homotopy spheres, Ann. of Math. 77 (1963), 504-537.

7. R. Lashof, Some theorems of Browder and Novikov on homotopy equivalent manifolds with an application, University of Chicago, Notes.

8. J. Milnor, A procedure for killing the homotopy groups of differentiable manifolds, Proc. Sympos. Pure Math., Vol. III, Amer. Math. Soc., Providence, R. I., 1961, pp. 39-55.

9. —_ On manifolds homeomorphic to the 7-sphere, Ann. of Math. 64 (1956), 399-405.

10. J. Munkres, Elementary differential topology, Princeton Univ. Press, Princeton, N. J., 1963.

11. _- Obstructions to the smoothing of piecewise differentiable homeomorphisms, Ann. of Math. 72 (1960), 521-554.

12. S. Novikov, Homotopically equivalent smooth manifolds. I, Izv. Akad. Nauk SSSR Ser. Mat 28 (1964), 365-474; English transl., Amer. Math. Soc. Transl. (2) 48 (1965), 271-396.

13. S. Smale, Differentiable and combinatorial structures on manifolds, Ann. of Math. 74 (1961), 498-502.

14. N. Steenrod, The topology of fibre bundles, Princeton Univ. Press, Princeton, N. J., 1951.

15. D. Sullivan, On the Hauptvermutung for manifolds, Bull. Amer. Math. Soc. 73 (1967), 598-600.

16. R. Thom, Quelques propriétés globales des variétés différentiables, Comment. Math. Helv. 28 (1954), 17-87.

17. J. Wagoner, Surgery on a map and an approach to the Hauptvermutung, Thesis, Princeton University, Princeton, N. J., 1966.

18. C. T. C. Wall, An extension of results of Novikov and Browder, Amer. J. Math. 88 (1966), 20-32.

UNIVERSITY OF MASSACHUSETTS, AMHerst, MassachusetTS 